Revue des patrimoines

28 | 2016

Le moulage. Pratiques historiques et regards contemporains

\title{
Ces traces qui nous parlent. Étude technique de la collection des épreuves en plâtre de la villa Médicis
}

Traces which speak to us. A technical study of the collection of plaster models at the Villa Médicis

\section{Pascale Roumégoux}

\section{(2) OpenEdition}

Journals

Édition électronique

URL : http://journals.openedition.org/insitu/12613

DOI : $10.4000 /$ insitu. 12613

ISSN : $1630-7305$

Éditeur

Ministère de la culture

Référence électronique

Pascale Roumégoux, "Ces traces qui nous parlent. Étude technique de la collection des épreuves en plâtre de la villa Médicis », In Situ [En ligne], 28 | 2016, mis en ligne le 15 mars 2016, consulté le 01 mai 2019. URL : http://journals.openedition.org/insitu/12613 ; DOI : 10.4000/insitu.12613

Ce document a été généré automatiquement le 1 mai 2019.

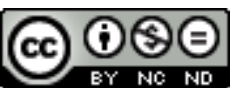

In Situ Revues des patrimoines est mis à disposition selon les termes de la licence Creative Commons Attribution - Pas d'Utilisation Commerciale - Pas de Modification 4.0 International. 


\title{
Ces traces qui nous parlent. Étude technique de la collection des épreuves en plâtre de la villa Médicis
}

\author{
Traces which speak to us. A technical study of the collection of plaster models at \\ the Villa Médicis
}

Pascale Roumégoux

Je tiens à remercier très sincèrement de leur soutien lors de cette année de résidence : Éric de Chassey, Jean-Luc Martinez, Alexandre Maral, Annick Lemoine, Alessandra Gariazzo, Pauline Pernet, Didier Repellin, Francoise Laurent, Antoinette Le Normand-Romain, Catherine Chevillot, Claire Barbillon, Élisabeth Le Breton, Colette Di Matteo, Hélène Palouzié, les restaurateurs de la société De Cesaris, Antoine Amarger, Guy Devreux, Camille Mathieu, Arielle Lebrun, Jacques Bourgeois et Béatrice Kostner.

Contribution complémentaire aux Actes des journées d'étude « Le moulage. Pratiques historiques, regards contemporains » (Cité de l'architecture et du patrimoine/musée du quai Branly, 14 \& 15 novembre 2012).

1 Cet article est la synthèse d'une étude réalisée sur la collection des tirages ${ }^{1}$ en plâtre conservée au sein de l'Académie de France à Rome, à la villa Médicis². Cette recherche

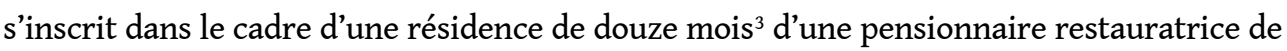
sculptures, amenée à intervenir régulièrement sur les collections de tirages de diverses institutions françaises ${ }^{4}$.

Centrée principalement sur l'observation de la matière, son mode de mise en œuvre et l'analyse des traces lisibles à la surface des épreuves, cette étude aspire initialement à approfondir la compréhension technique de ces œuvres, et à mieux appréhender leur procédé de moulage et de tirage ${ }^{5}$. L'analyse des informations recueillies ${ }^{6}$ a permis à la restauratrice de poser un regard nouveau sur ces témoins de l'Histoire au parcours 
souvent complexe, et vise à alimenter le champ des investigations menées sur cette collection?

\section{La collection de la Villa Médicis}

La villa Médicis conserve aujourd'hui trois cent soixante-dix-neuf tirages en plâtre qui illustrent majoritairement les grands chefs-d'œuvre de l'art antique, dont les marbres originaux sont actuellement présentés dans les grands musées du monde entier. Quelques reproductions d'œuvres médiévales, importées de France, et des créations des sculpteurs pensionnaires complètent cet ensemble.

Figure 1

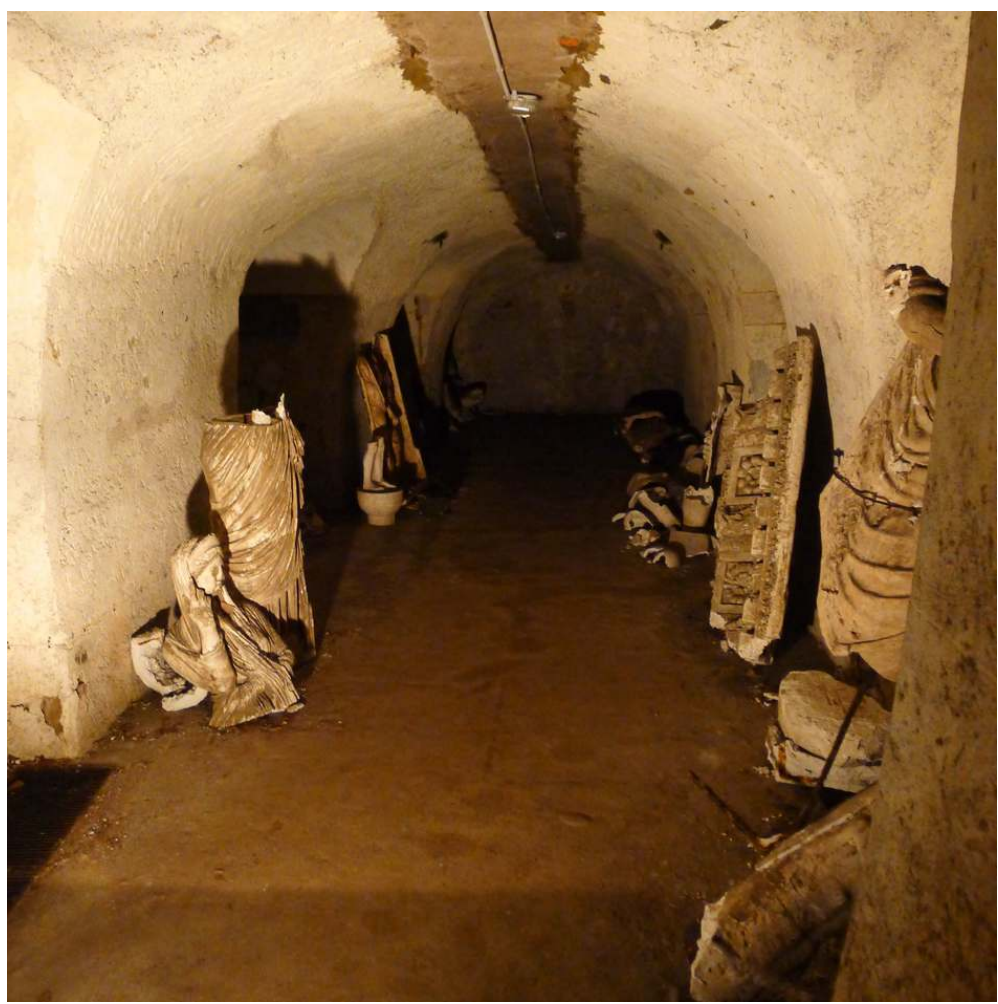

Les œuvres installées dans les caves sous la terrasse du Bosco de la villa Médicis, octobre 2012. Phot. Roumégoux, P. (c) Pascale Roumégoux.

Dès 1684, ces épreuves en plâtre, supports d'étude et d'inspiration, occupent une place privilégiée au sein de l'Académie de France à Rome ${ }^{8}$. Transférée à Rome dans les divers lieux successivement occupés par l'Académie, puis en partie dispersée entre Rome et Naples à la fin du XVIII ${ }^{e}$ siècle, la collection est rassemblée à partir de 1804 à la villa Médicis. Complétée, elle est installée dans la « Grande Galerie » et dans plusieurs salles de la villa9 . Placée au centre du bâtiment et de l'intérêt des artistes durant des décennies, elle tombe en disgrâce sous le directorat du peintre Balthus durant les années soixante ${ }^{10}$. Plusieurs centaines d'œuvres sont alors reléguées dans des caves humides situées sous le Bosco de la villa et y resteront durant plus de quarante ans (fig. 1). En 2009, une partie de cette collection est sauvée de l'oubli et installée dans la gypsothèque pour être présentée au public $^{11}$ (fig. 2). Puis, c'est grâce à la persévérance de plusieurs spécialistes ${ }^{12}$ conscients de la valeur de cette collection que plus de cent quatre-vingt-cinq tirages 
rescapés sont installés à l'abri dans une réserve aménagée à la fin de l'année 2013, située dans l'ancienne menuiserie de l'Académie (fig. 3).

Figure 2

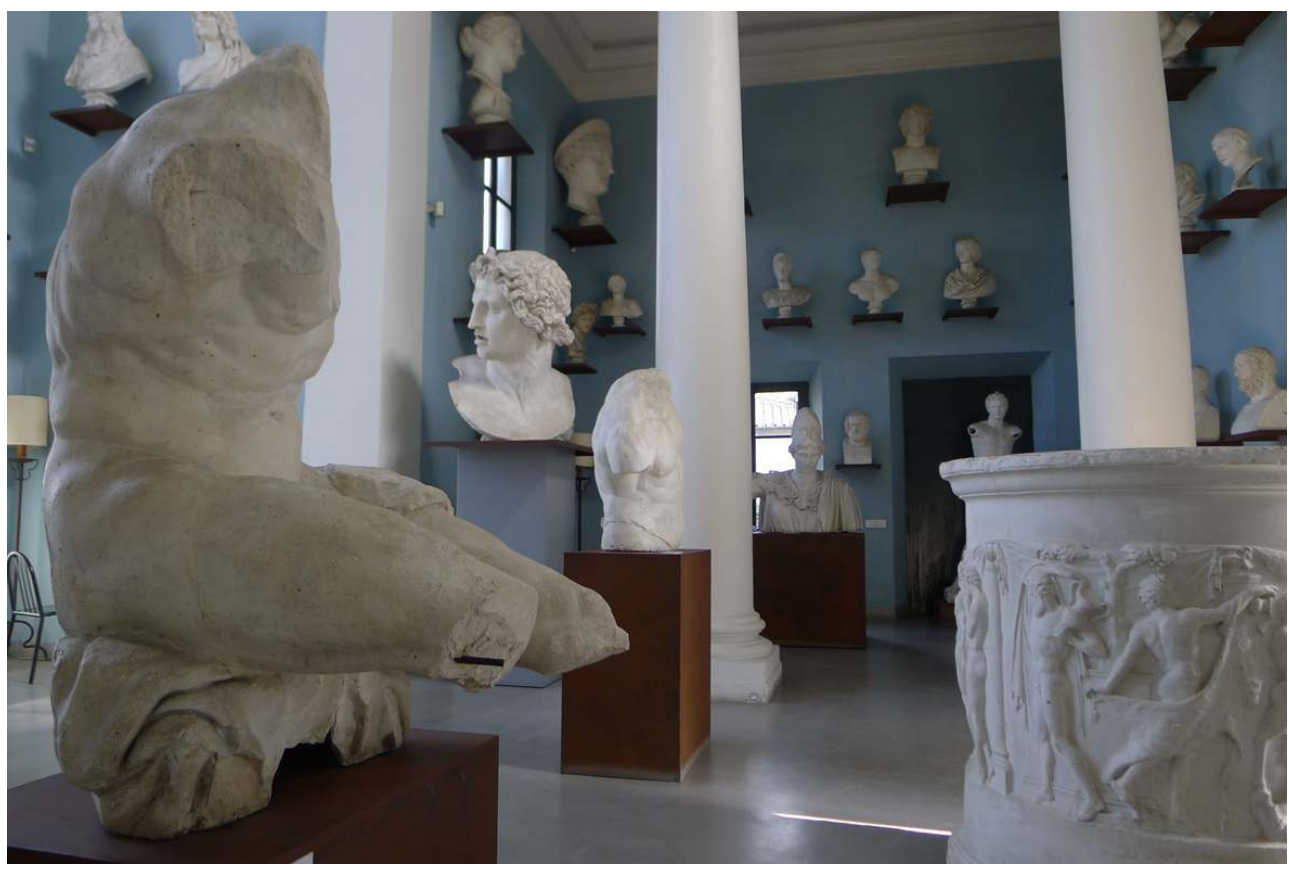

La gypsothèque de la villa Médicis.

Phot. Roumégoux, P. ( ) Pascale Roumégoux.(C) 
Figure 3

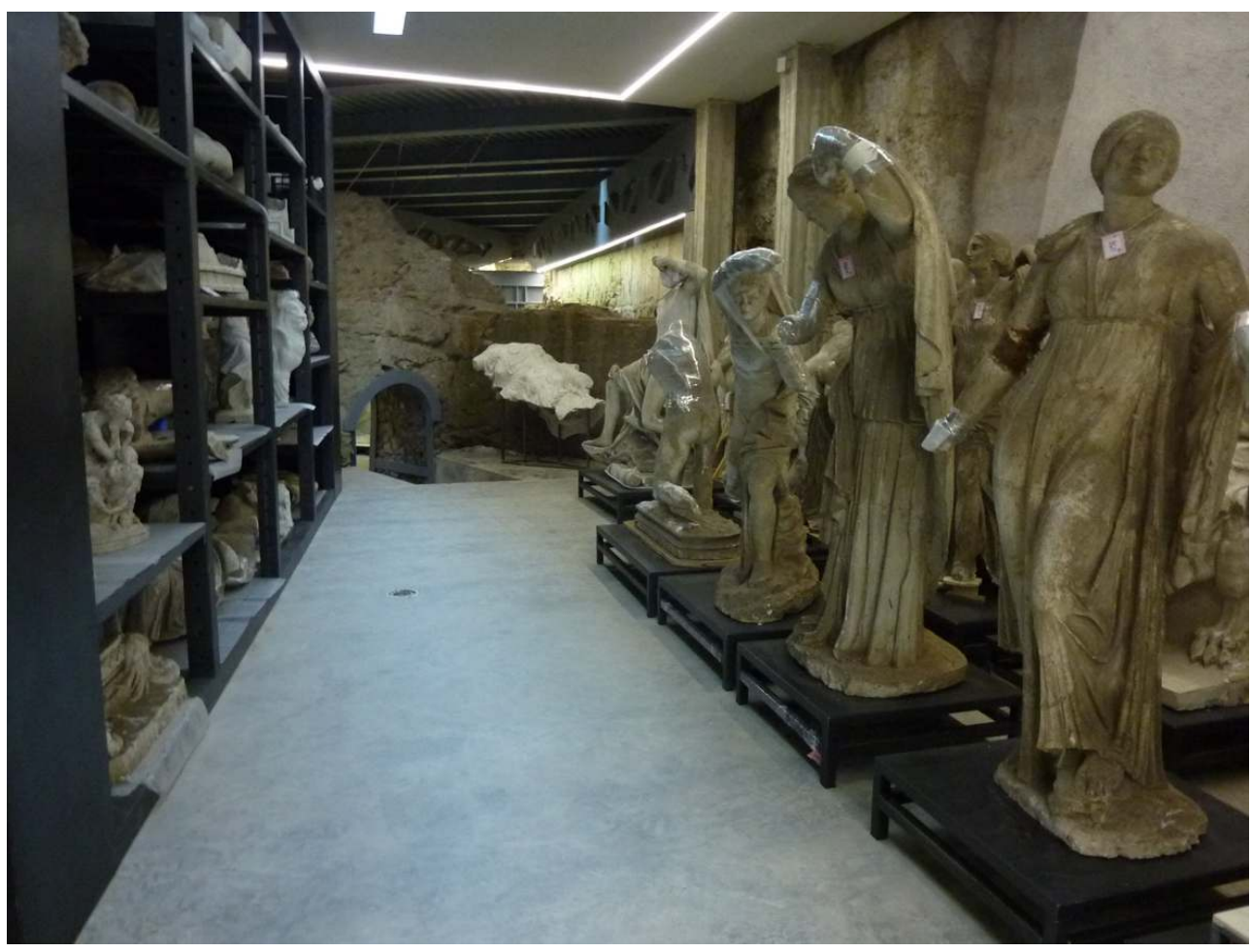

Nouvelle réserve des tirages en plâtre de la villa Médicis, janvier 2014.

Phot. Roumégoux, P. (c) Pascale Roumégoux.

Enfin accessible, cette collection dévoile peu à peu toute sa richesse d'enseignements sur le plan technique et historique.

6 Procéder à une telle étude sur un corpus d'œuvres dans son intégralité offre le grand avantage de permettre une confrontation immédiate des tirages et de leurs caractéristiques techniques. Au fur et mesure de l'avancée des investigations, des ensembles semblent se dessiner, des comparaisons s'imposent, contribuant à donner une meilleure compréhension. Parfois même, l'accumulation d'informations incomplètes, mais récurrentes, permet d'éclairer les observations. C'est par exemple le cas pour les tirages des Niobides (fig. 4), dont la surface a été partiellement dissoute lors d'une exposition en extérieur, ou pour les reliefs de la colonne Trajane ${ }^{13}$ (fig. 5) datés du XVII siècle ${ }^{14}$, qui présentent une surface parfois très usée. Une histoire chaotique, associée à d'anciennes « restaurations » inadaptées ${ }^{15}$, entrave parfois la bonne lisibilité des traces techniques superficielles mais grâce à la récurrence des observations, des hypothèses quant aux procédés de moulage ont pu être élaborées. 
Figure 4

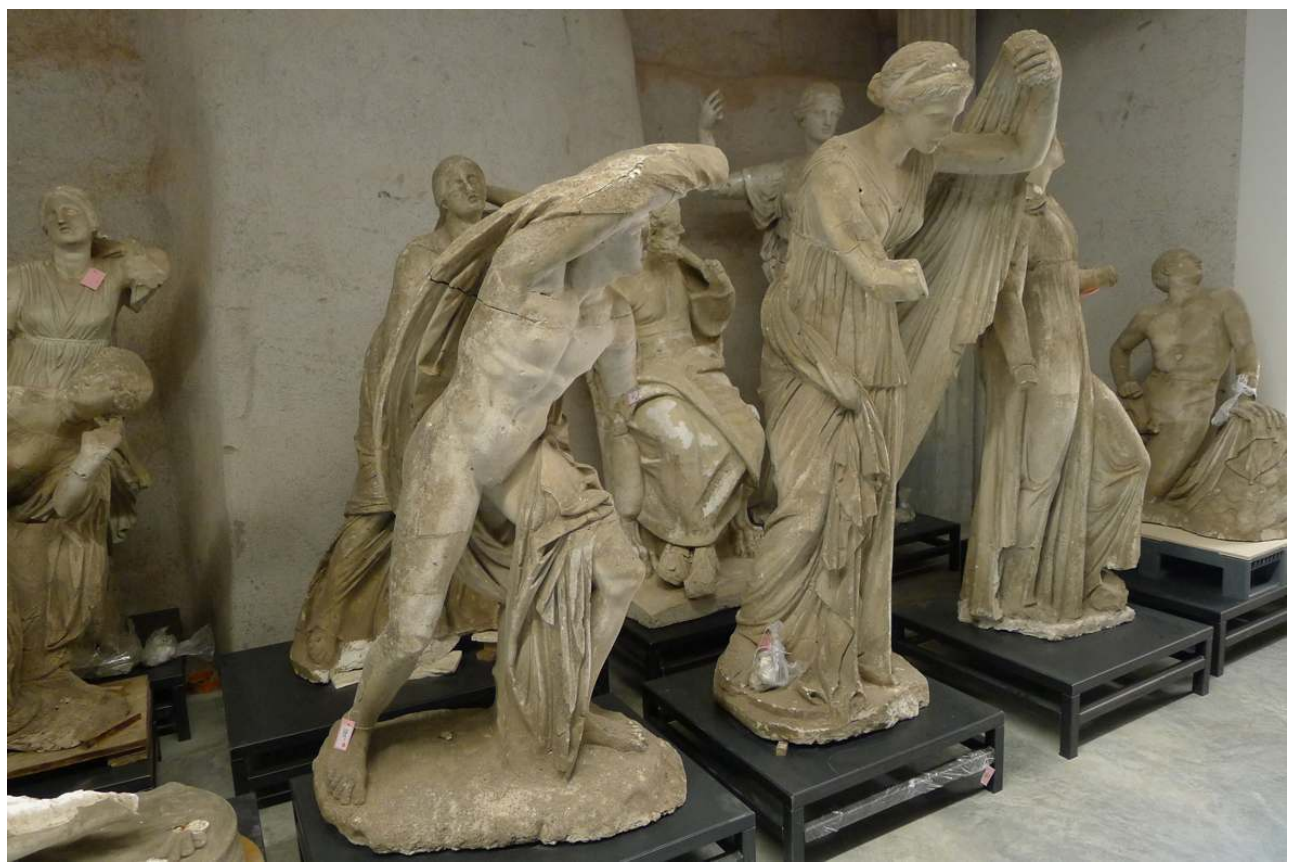

Tirages des Niobides conservés dans la réserve des tirages de la villa Médicis. Phot. Roumégoux, P. (c) Pascale Roumégoux.

\section{Figure 5}

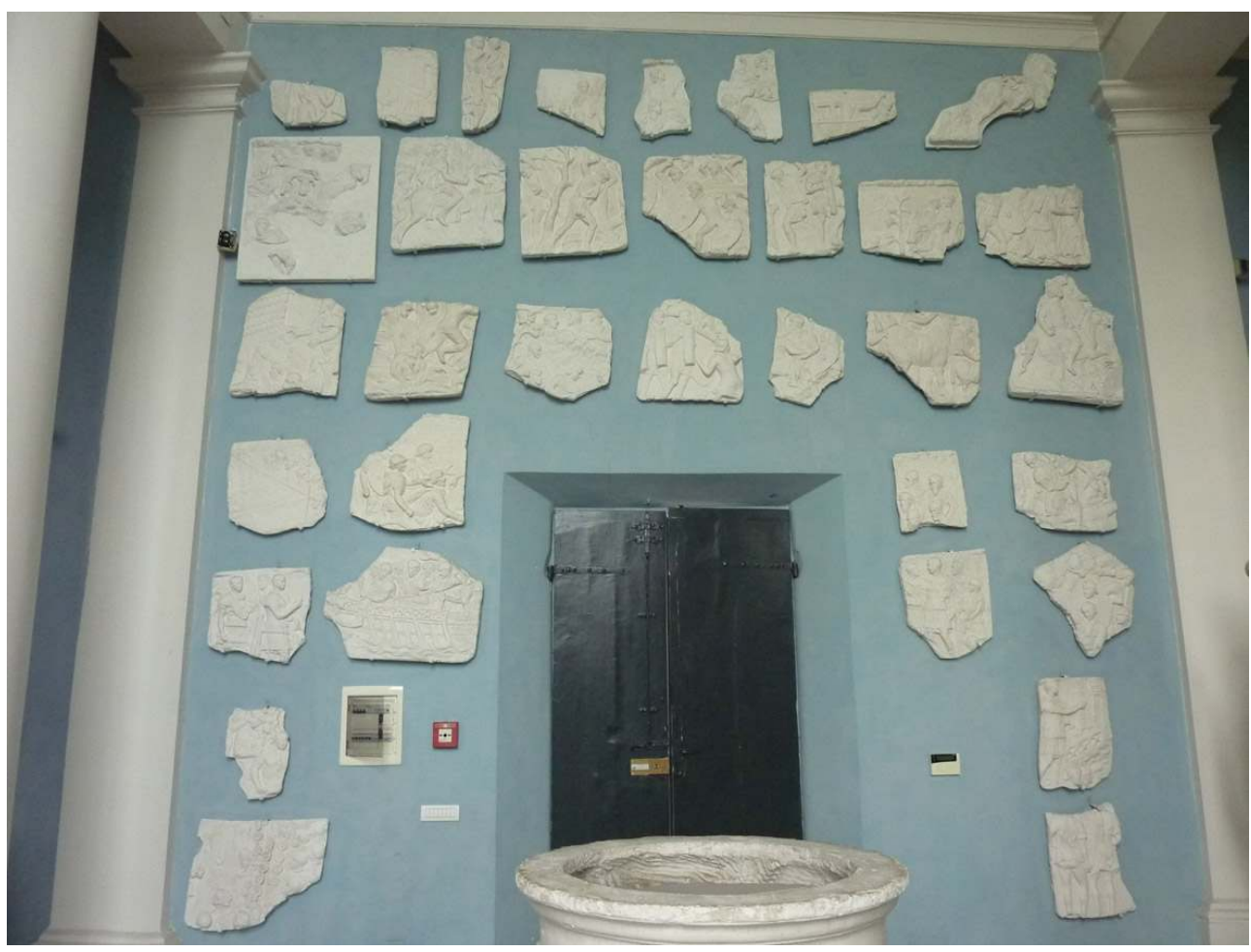

Les tirages de la colonne Trajane, conservés dans la gypsothèque de la villa Médicis.

Phot. Roumégoux, P. (c) Pascale Roumégoux. 


\section{La matière au centre de l'étude}

$7 \quad$ L'analyse des procédés techniques de mise en œuvre de ces tirages s'est appuyée dans un premier temps sur une observation de l'œuvre dans sa globalité ${ }^{16}$. L'état fragmentaire de cette collection permet de visualiser l'intérieur des épreuves, et ainsi de renseigner le mode de tirage. La localisation des armatures, la détermination de leur nature - en fer, en fer forgé pour les plus anciennes, en bois ou plus rarement en os - la présence de filasse ${ }^{17}$ et l'épaisseur des tirages sont des informations précieuses facilitant la compréhension de cet ensemble.

8 L'étude du plâtre constitutif a également été au centre de l'attention: sa couleur, sa granulométrie, son mode de gâchage ${ }^{18}$ et de mise en œuvre. Ces données sont importantes, notamment pour la définition des ensembles évoqués précédemment, et développés un peu plus tard dans cet exposé.

Par l'observation attentive de la surface de ces tirages, on peut également étudier les coutures, vestiges du procédé de moulage ${ }^{19}$. Ces traces en faible relief matérialisent les contours des différentes pièces qui composent le moule ${ }^{20}$, duquel a été tirée l'épreuve. L'analyse combinée du profil des coutures, de leur nombre et de leur localisation aide à estimer quelle était la technique de moulage utilisée: moule à bon-creux à pièces en plâtre, moule estampé à la terre crue, moule à creux-perdu ${ }^{21}$ entre autres.

\section{Les techniques de moulage}

10 Cette étude révèle qu'une importante partie des tirages de la collection italienne semble issue de moules à bon-creux à pièces en plâtre ${ }^{22}$, technique de moulage exigeante et onéreuse ${ }^{23}$. Les coutures observées à la surface de ces tirages présentent toutes un profil similaire : des arêtes assez fines et régulières délimitant des pièces aux formes simples, idéalement à angle droit, souvent nombreuses. Ainsi, le réseau de coutures formé est généralement dense, tel qu'on l'observe sur le tirage partiel de la Pallas de Velletri ${ }^{24}$ conservé à la gypsothèque (fig. 6). Les coutures sont positionnées en tenant compte précisément des formes en contre-dépouille ${ }^{25}$. Ces moules pérennes permettent de tirer de nombreuses épreuves, d'où la nécessité de confectionner des pièces aux formes simples, peu fragiles. Suite à un usage répétitif, les moules peuvent s'altérer et ainsi laisser à la surface des tirages des coutures au profil plus large et irrégulier. L'aspect déformé de ces coutures peut alors être confondu avec un réseau de coutures d'un tirage issu d'un estampage. 
Figure 6

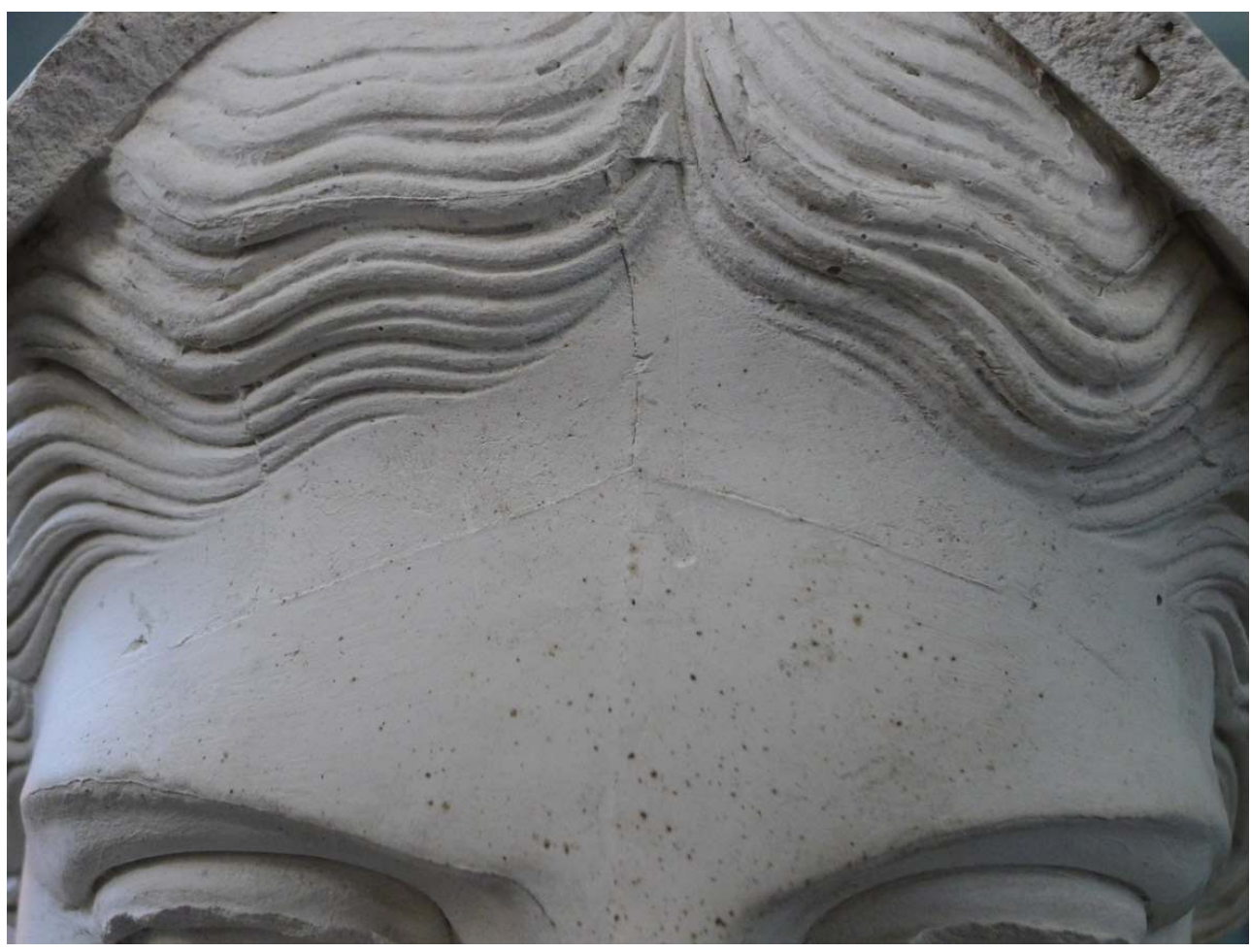

Coutures visibles à la surface du tirage de la Pallas de Velletri, conservé dans la gypsothèque de la villa Médicis.

Phot. Roumégoux, P. (c) Pascale Roumégoux.

11 En effet, d'après les observations, les moules estampés à la terre crue génèrent un réseau de coutures dont la crête est ondulante, légèrement irrégulière. Les coutures peuvent être fines, mais sont généralement plus larges que les coutures issues d'un moule à pièces en plâtre. Leur largeur dépend de la grandeur de la pièce confectionnée : plus celle-ci est étendue, plus le retrait lors de la dessiccation de la terre est important et plus la couture sera grossière ${ }^{26}$. Les pièces formées peuvent avoir une forme aux angles aigus comme sur le tirage partiel du Dioscure de Montecavallo ${ }^{27}$ (fig. 7). Elles sont généralement moins nombreuses, la souplesse de la terre offrant une certaine tolérance lors du démoulage sur les formes complexes ${ }^{28}$. Cependant, certaines œuvres de cette collection ont fait l'objet d'une attention particulière, tel que le tirage intégral de l'Hercule-Commode ${ }^{29}$ par exemple, qui présente un réseau de coutures dense, notamment au niveau du visage et de la chevelure. En résulte une prise d'empreinte de qualité. 
Figure 7

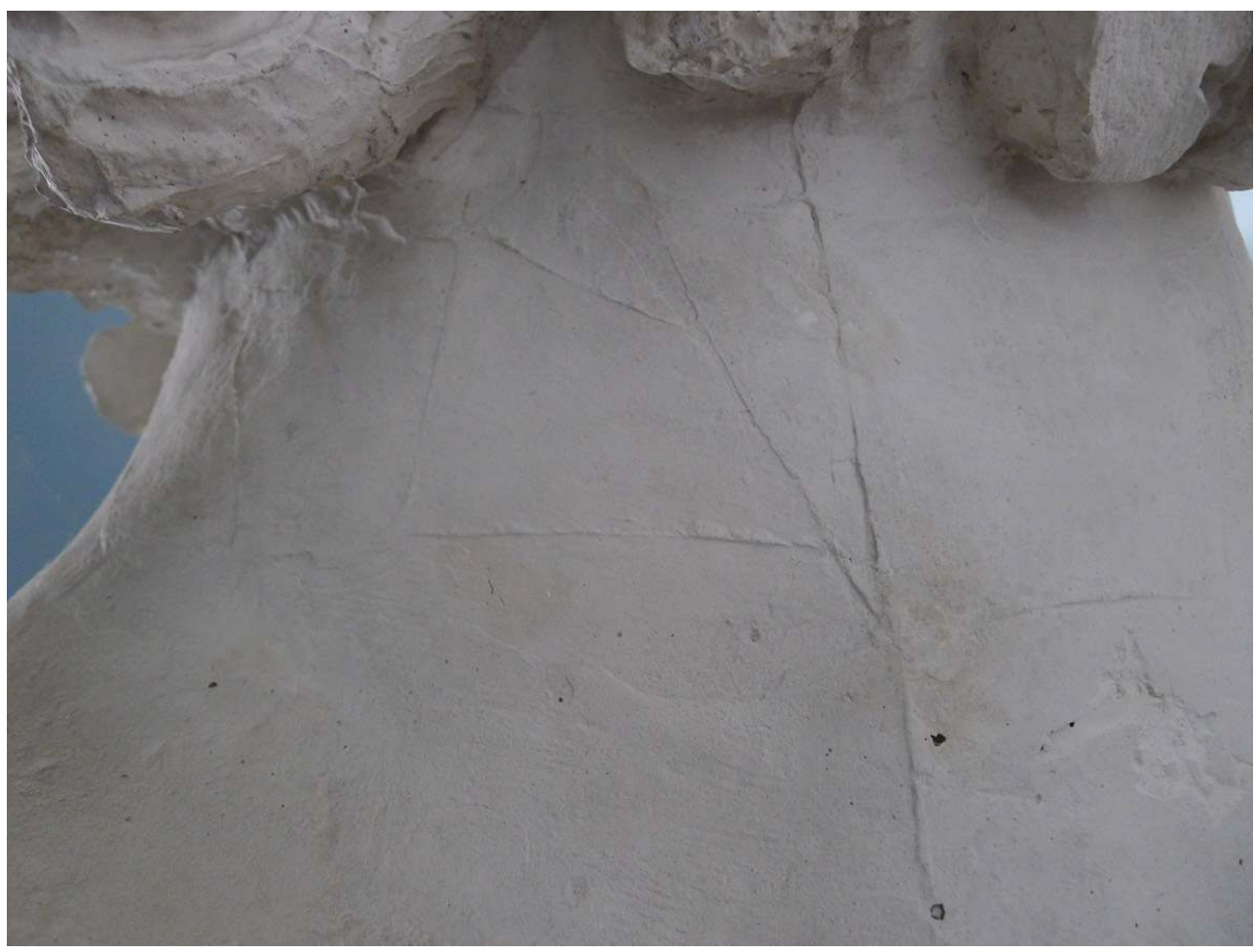

Coutures et pièces anguleuses sur le tirage partiel du Dioscure de Montecavallo, conservé dans la gypsothèque de la Villa Médicis.

Phot. Roumégoux, P. (c) Pascale Roumégoux.

12 Des décalages à la jonction des pièces sont souvent observés sur les tirages issus d'estampage, ainsi que des aspérités de surface très particulières ${ }^{30}$. La littérature ancienne indique que le nombre d'épreuves tirées dans ce type de moule est limité à deux ou trois maximum ${ }^{31}$. Inévitablement, la fidélité de l'empreinte, très fragile, s'amoindrit à chaque tirage : les détails sont moins bien rendus, les creux moins marqués, les décalages plus importants et les coutures souvent plus larges. Ceci pourrait expliquer la qualité d'empreinte moyenne des reliefs de la colonne Trajane réalisés lors de la campagne du $\mathrm{XVII}^{\mathrm{e}}$ siècle (fig. 8). S'agirait-il d'épreuves issues d'un deuxième ou troisième tirage? 
Figure 8

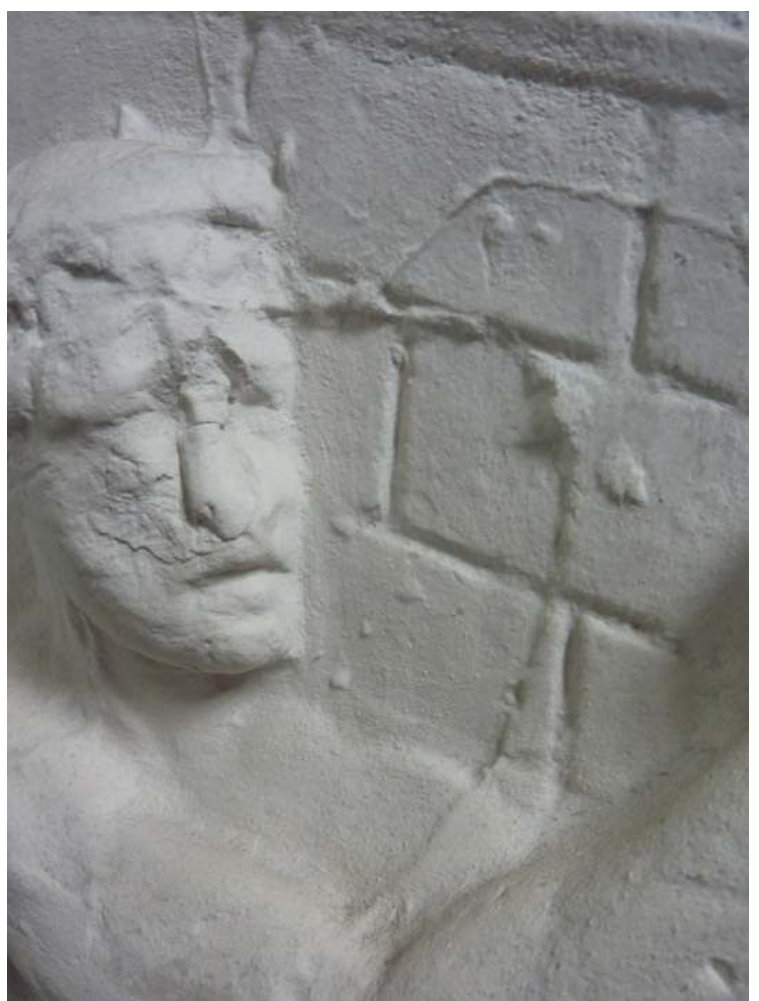

Coutures et décalages visibles à la surface du tirage de la plaque LIII, scène 132 - 133 de la colonne Trajane, daté du XVIle siècle et conservé dans la gypsothèque de la villa Médicis.

Phot. Roumégoux, P. (c) Pascale Roumégoux.

De plus, du fait de la dessiccation de la terre, l'utilisation de tels moules est limitée dans le temps. Ce procédé de moulage, ardu techniquement mais bien moins coûteux que la réalisation d'un moule à bon-creux en plâtre ${ }^{32}$, a largement été employée pour la collection de la villa, tant pour la réalisation de bas-reliefs que d'œuvres en pied $^{33}$.

L'emploi combiné de terre et de plâtre, définissant une technique mixte, semble également avéré sur plusieurs œuvres de la collection telles que le tirage intégral d' Anacréon $^{34}$ (fig. 9) exposé dans la gypsothèque. Dans ce cas, les pièces en terre, « parées ${ }^{35}$ » en premier, permettent de réaliser l'empreinte des parties complexes telles que la chevelure ou la barbe. Les parties plus étendues, de forme plus simple, sont moulées au plâtre, ce qui facilite la manipulation du moule. 
Figure 9

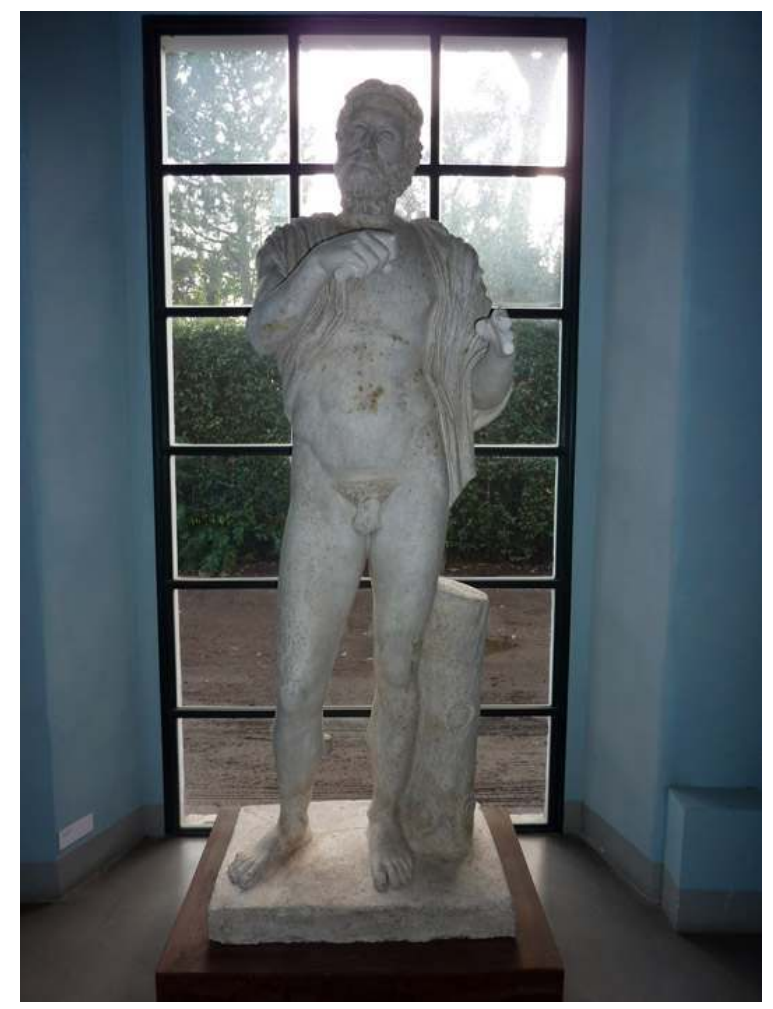

Tirage intégral d'Anacréon, conservé dans la gypsothèque de la villa Médicis.

Phot. Roumégoux, P. @ Pascale Roumégoux.

Il n'est pas exclu de trouver aussi dans cette collection une empreinte prise en partie ou intégralement avec un mastic cireux ${ }^{36}$. Ce procédé pourrait être combiné avec l'utilisation de plâtre. Son identification est cependant plus difficile. La question se pose pourtant pour la réalisation des bas-reliefs de la colonne Trajane durant la campagne du XIX ${ }^{\mathrm{e}}$ siècle. Ceux-ci présentent des coutures fines, légèrement ondulantes délimitant des pièces plus nombreuses que sur les tirages $d u \mathrm{XVII}^{\mathrm{e}}$ siècle. Ce matériau, contraignant à mettre en œuvre et pouvant s'avérer préjudiciable s'il est appliqué sur une surface altérée ${ }^{37}$, a pu cependant être sélectionné pour sa souplesse, sa grande fidélité d'empreinte et sa stabilité. L'emploi d'un moule de technique mixte (terre/plâtre) est également possible pour ces œuvres.

Enfin, un dernier procédé de moulage a pu être identifié sur environ une vingtaine d'épreuves : le moulage à creux-perdu. Ce procédé de moulage diffère des précédents de par la nature même du modèle à mouler. Réalisé sur un modèle généralement en terre, le moule doit être détruit pour extraire l'unique épreuve en plâtre. Ce procédé de moulage a été utilisé pour pérenniser les œuvres modelées par les sculpteurs durant leur séjour à l'Académie de France à Rome. Certaines de ces épreuves originales sont signées, telles que le portrait de Ziem par Ségoffin ${ }^{38}$ par exemple, ou facilement reconnaissable comme l'esquisse d'Ugolin et ses fils ${ }^{39}$ de Carpeaux (fig. 10). Ces moules sont généralement composés de deux parties pour les bustes ou d'un nombre limité de pièces pour les œuvres en pied comme Ugolin et ses fils. 
Figure 10

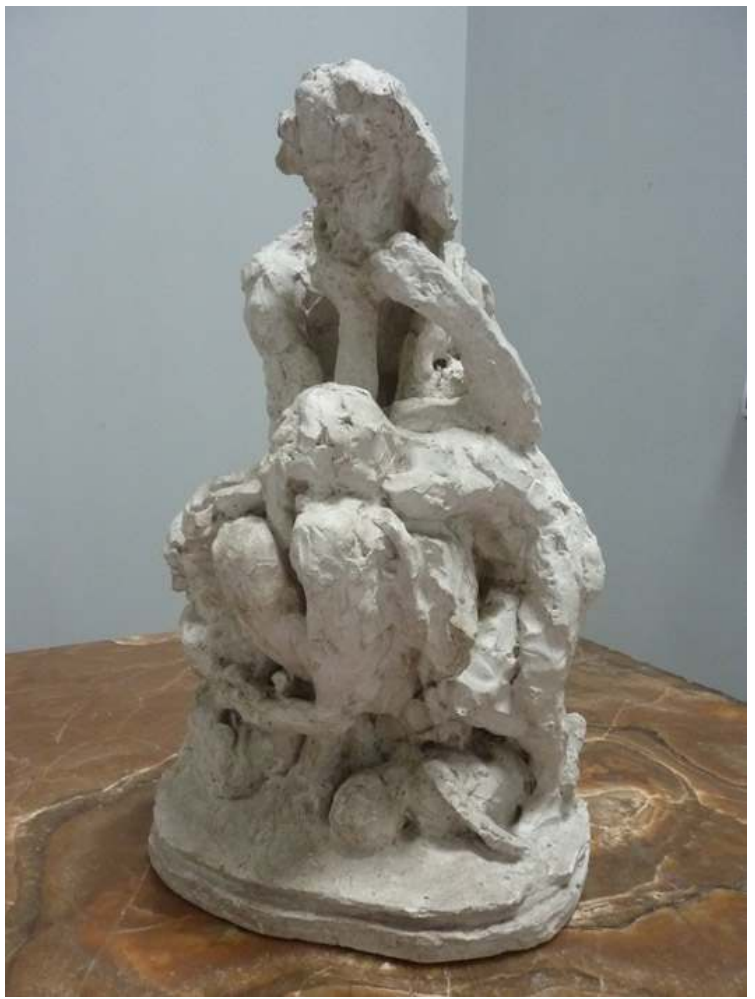

Tirage original d'une esquisse d'Ugolin et ses fils de Carpeaux, XIX ${ }^{\mathrm{X}}$ siècle. Phot. Roumégoux, P. (c) Pascale Roumégoux.

\section{Autres informations collectées sur la surface des œuvres}

17 L'étude des coutures peut également renseigner «l'origine» d'un tirage. Ainsi, l'observation d'un double réseau de coutures, c'est-à-dire de deux coutures souvent parallèles, localisées généralement dans la même zone, permet d'identifier un "surtirage ", tirage réalisé d'après une autre épreuve en plâtre ${ }^{40}$. Ainsi, les deux réseaux observés proviennent l'un du premier tirage moulé et l'autre de la deuxième prise d'empreinte (fig. 11). Un double réseau a pu être observé sur plus de quarante-cinq œuvres de la villa Médicis ${ }^{41}$. Des liens entre les tirages au sein même de la collection ont pu être établis : des épreuves ont été moulées d'après des tirages existants, multipliant les modèles. En attestent les nombreuses incisions observées sur plus de quatre-vingt-dix œuvres. Ces traces en creux sont laissées par les couteaux des mouleurs qui réalisent les prises d'empreinte en plâtre sur les tirages ${ }^{42}$. Ces mêmes traces sont parfois identifiées à la surface des œuvres originales en pierre. Ces incisions s'accompagnent souvent de restes d'agents de séparation, couche protectrice qui permet d'isoler le modèle avant la prise d'empreinte. L'identification de ces restes d'agent de démoulage, de type barbotine ${ }^{43}$ ou savon noir, par exemple, constitue une information intéressante pour comprendre les techniques employées. Ceci prouve combien cette collection, en perpétuelle évolution, était avant tout un outil de travail pour les pensionnaires, renouvelé, complété ou déplacé 
en fonction des besoins ${ }^{44}$. Ceci témoigne également de la difficulté de définir avec certitude l'origine d'un tirage.

Figure 11

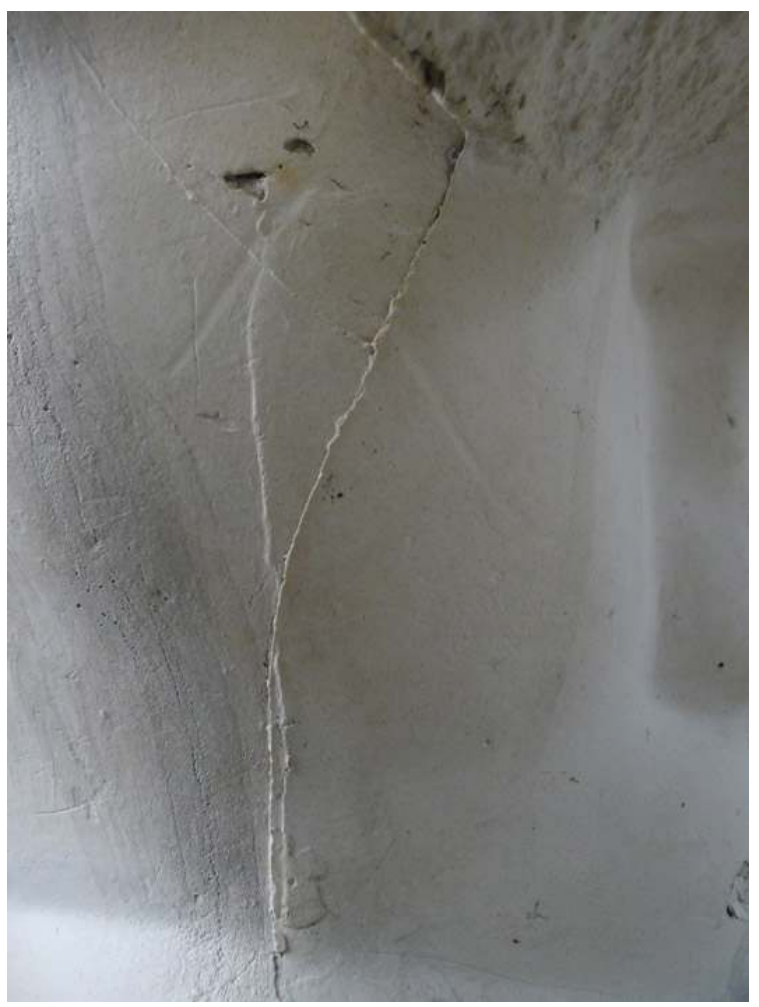

Double réseau de coutures visible à la surface du tirage du Torse de satyre, conservé dans la gypsothèque de la villa Médicis.

Phot. Roumégoux, P. @ Pascale Roumégoux.

18 A contrario, certains tirages, datés principalement des $\mathrm{XIX}^{\mathrm{e}}$ et $\mathrm{xx}^{\mathrm{e}}$ siècles, portent des estampilles qui renseignent clairement le nom du mouleur ou de l'atelier de moulage. Ainsi, l'estampille du mouleur italien Leopoldo Maipieri est observée sur le tirage intégral du Démosthène ${ }^{45}$ daté de la première moitié du xix ${ }^{e}$ siècle. Sont également visibles les estampilles de divers ateliers de moulage français : "Musée d'Angers », « Musée Royal », « Musées nationaux moulages », ou encore « Musée du Trocadéro ». À l'exception de deux d'entre eux, la totalité de ces tirages est importée de France. À cela s'ajoutent des inscriptions telles que « Musée Capitolino » inscrite sur la terrasse d'un Hercule Enfant ${ }^{46}$ ou celle «M.S.C. $1919 »^{47}$ (musée de Sculpture comparée) visible au revers d'œuvres médiévales (fig. 12). 


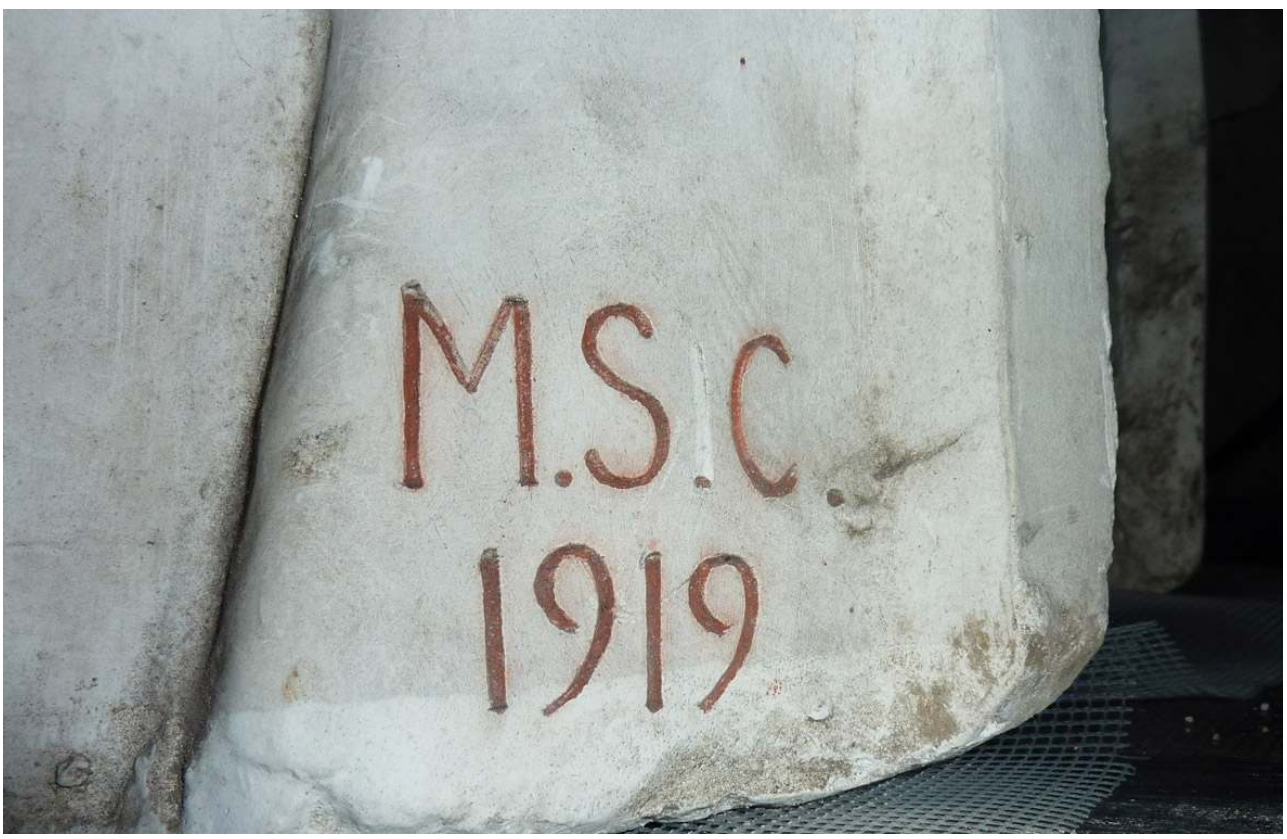

Inscription « MSC » indiquant le musée de sculpture comparée.

Phot. Roumégoux, P. (c) Pascale Roumégoux.

Enfin, d'autres éléments caractéristiques de l'utilisation de cette collection ont pu être relevés à la surface de plus d'une quarantaine d'œuvres : des clous en bronze (fig. 13), des points de basement en fer et des croix inscrites à la mine de plomb. Ces marques sont les vestiges de la technique de taille indirecte à la mise aux points. L'utilisation des «trois compas » ou d'une machine à mettre aux points - outil inventé au cours du XIX ${ }^{\mathrm{e}}$ siècle permettait de réaliser des copies en pierre d'après les modèles en plâtre ${ }^{48}$. Cet exercice, obligatoire pour les pensionnaires sculpteurs ${ }^{49}$, visait à reproduire, parfois seulement dans les grandes lignes ${ }^{50}$, les chefs-d'œuvre de l'art antique. Cette technique était également utilisée pour réaliser en pierre les créations modelées par les pensionnaires, comme en attestent les clous en bronze retrouvés sur cinq tirages originaux de la collection. 


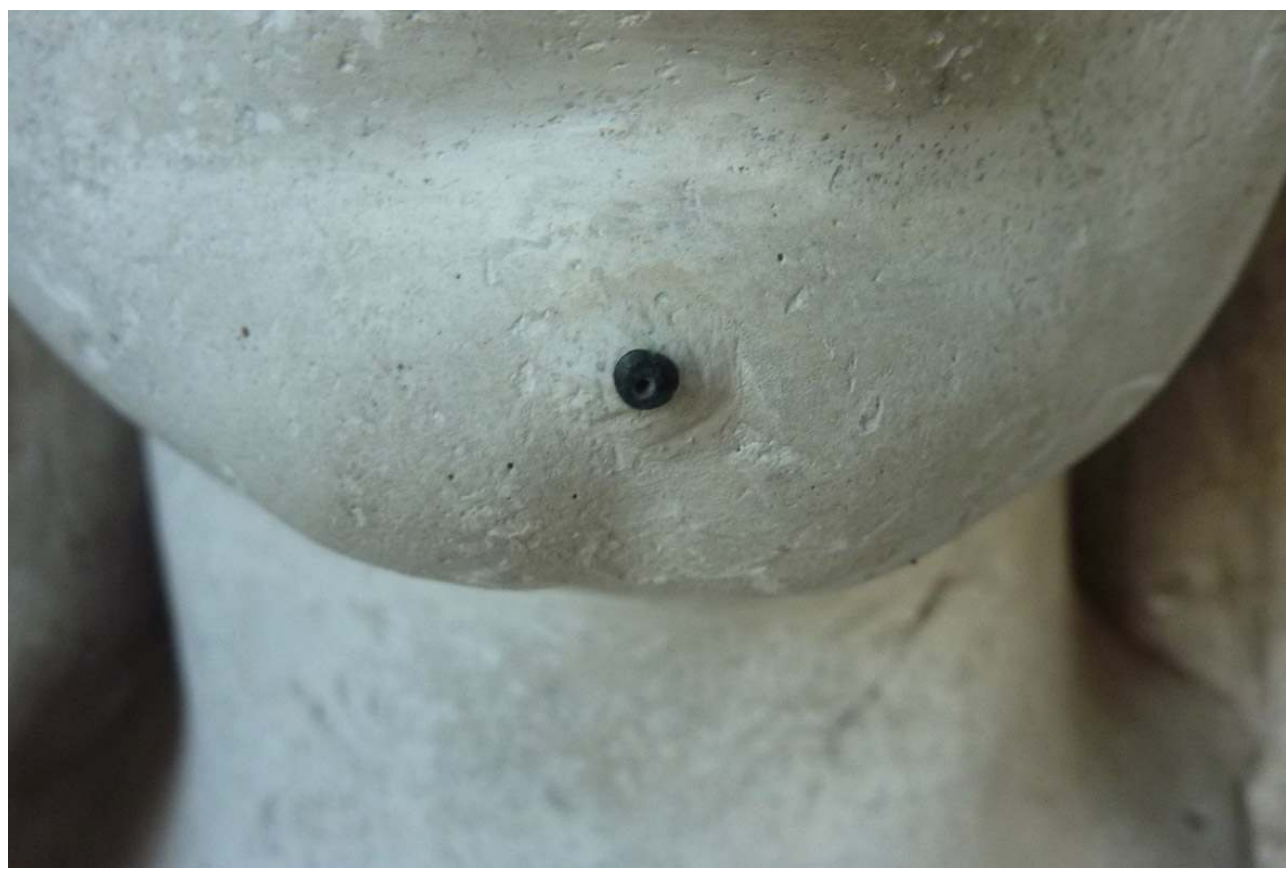

Clou en bronze pour la technique de mise-aux-points aux trois compas visible sur le tirage d'un buste féminin non identifié (NIP0299), conservé dans la gypsothèque de la villa Médicis.

Phot. Roumégoux, P. (c) Pascale Roumégoux.

\section{Les apports de l'étude et ses limites} épreuves mais également de soumettre des axes de recherche relatifs à leur histoire. Pour être pleinement exploitable, une étude technique doit s'inscrire dans le cadre d'une recherche globale, alimentée par des données historiques et stylistiques, par l'étude des archives et de la bibliographie ancienne.

21 Ainsi, la détermination d'un procédé de moulage peut-elle donner des indices de temps: dans le cas de moules à creux-perdu par exemple, le moule est souvent réalisé rapidement après le travail de modelage. Lorsque l'artiste est identifié, le tirage original obtenu est situé dans le temps. De même, l'identification de tirages issus de moules à pièces estampés en terre, ou de technique mixte, suggère des indications de temps et de lieu pour la prise d'empreinte et le tirage: en effet, les épreuves doivent être tirées rapidement dans ces moules délicats dont la conservation est limitée dans le temps. De fait, les tirages obtenus ont sans doute été réalisés dans un périmètre relativement proche des œuvres originales, souvent des chefs d'œuvres dont l'histoire est bien connue. Enfin, le type de moule conditionne également le nombre d'épreuves: unique pour un moule à creux-perdu ou limité à trois maximum pour les moules estampés.

A contrario, les moules à bon-creux à pièces en plâtre sont pérennes, déplaçables facilement et autorisent plusieurs tirages. 


\section{Des œuvres au profil singulier}

\section{Figure 14}

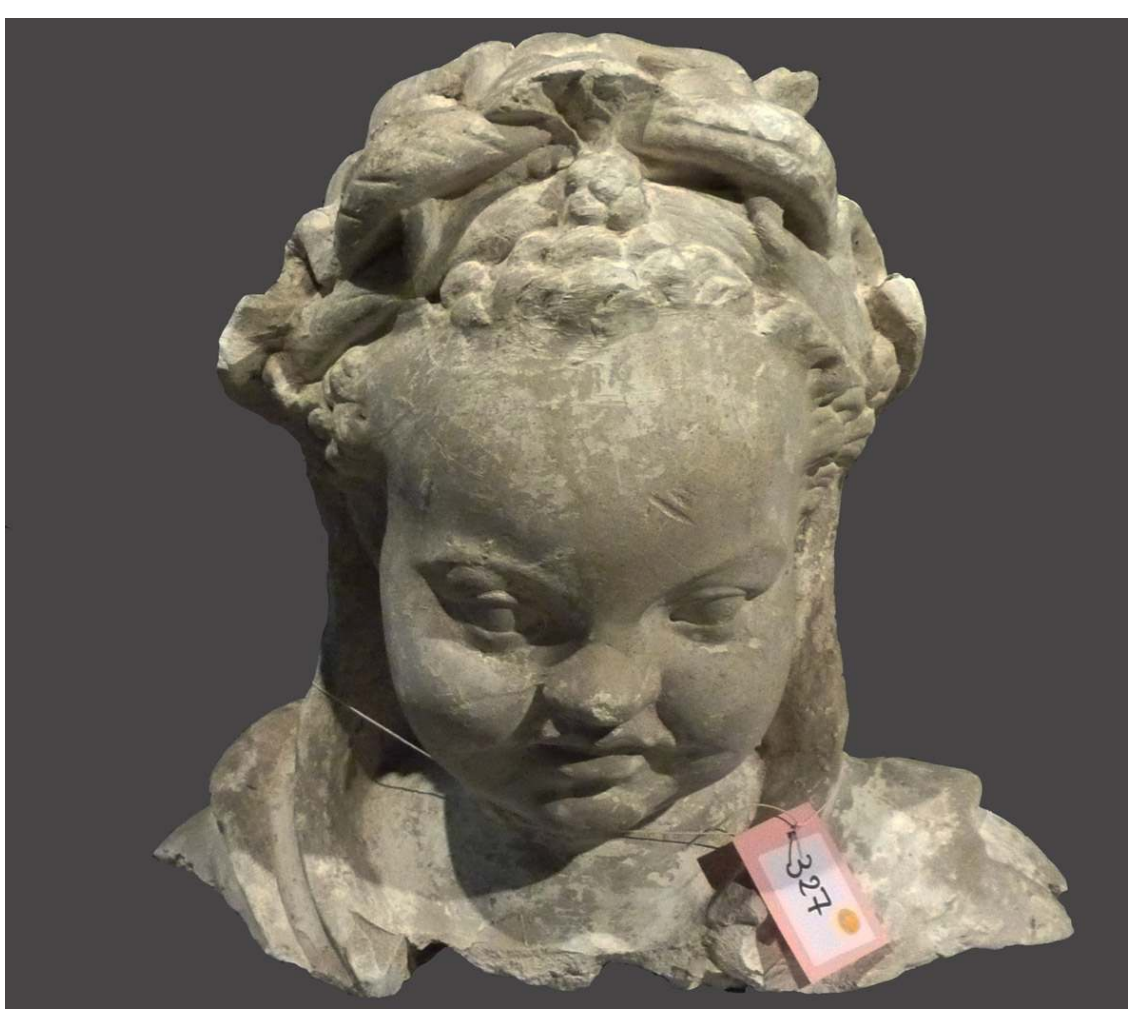

Tirage partiel de Hercule Enfant, conservé dans la réserve des tirages de la villa Médicis. Phot. Roumégoux, P. @ Pascale Roumégoux. 
Figure 15

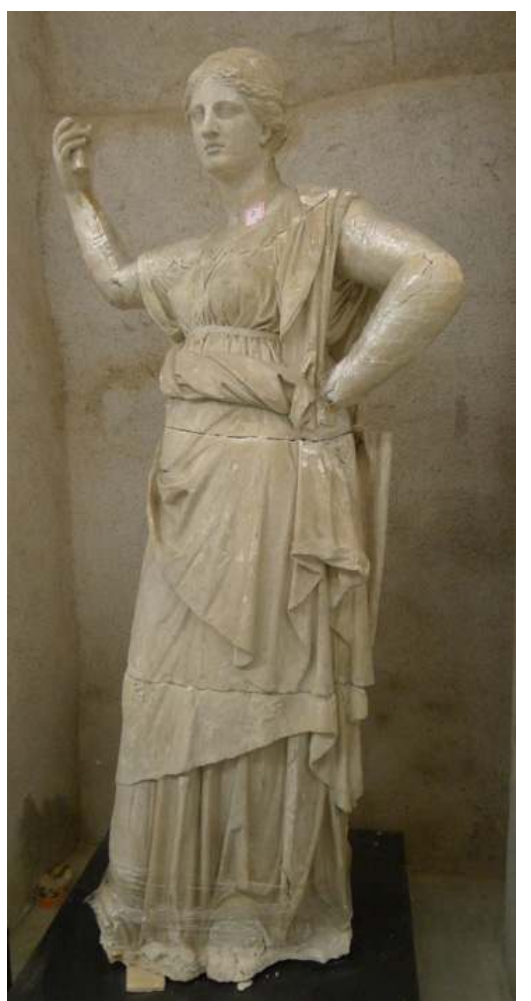

Tirage intégral de Junon Cesi, conservé dans la réserve des tirages de la villa Médicis.

Phot. Roumégoux, P. ( ) Pascale Roumégoux.

Les matériaux constitutifs des tirages sont également des sources d'informations précieuses. Leur nature singulière et leur mode de mise en œuvre incitent à considérer certaines épreuves de cette collection comme pouvant constituer un corpus à part. Ainsi, par exemple, une Tête d'Hercule enfant ${ }^{51}$ (fig. 14), les tirages intégraux de la Junon Cesi ${ }^{52}$ (fig. 15), de l'Apollon du Belvédère ${ }^{53}$ ou encore de l'Hercule-Commode déjà cité sont tous tirés avec un plâtre d'aspect grossier (fig. 16), mis en œuvre en épaisseur. Il présente de nombreuses bulles de surface, indiquant qu'il a été gâché clair, c'est-à-dire avec une quantité d'eau plus importante que la moyenne habituelle. En général, ce plâtre est appliqué en grande épaisseur, jusqu'à huit ou dix centimètres, générant des tirages au poids important. Ce plâtre grossier est très différent de celui employé pour les tirages datés $d u \mathrm{xIX}^{\mathrm{e}}$ et $\mathrm{du} \mathrm{xx}^{\mathrm{e}}$ siècle, beaucoup plus fin et dense. Il est exempt d'impuretés visibles à l'œil nu ${ }^{54}$ mais évoque une fabrication pouvant s'avérer ancienne ${ }^{55}$. Cependant, il convient de rester prudent dans l'interprétation. En effet, le mode de transformation et de préparation du plâtre conditionne son aspect, ses propriétés mécaniques et son mode de mise en œuvre; cependant, l'évolution du procédé de fabrication n'exclut pas la pratique concomitante d'anciennes techniques de préparation. 


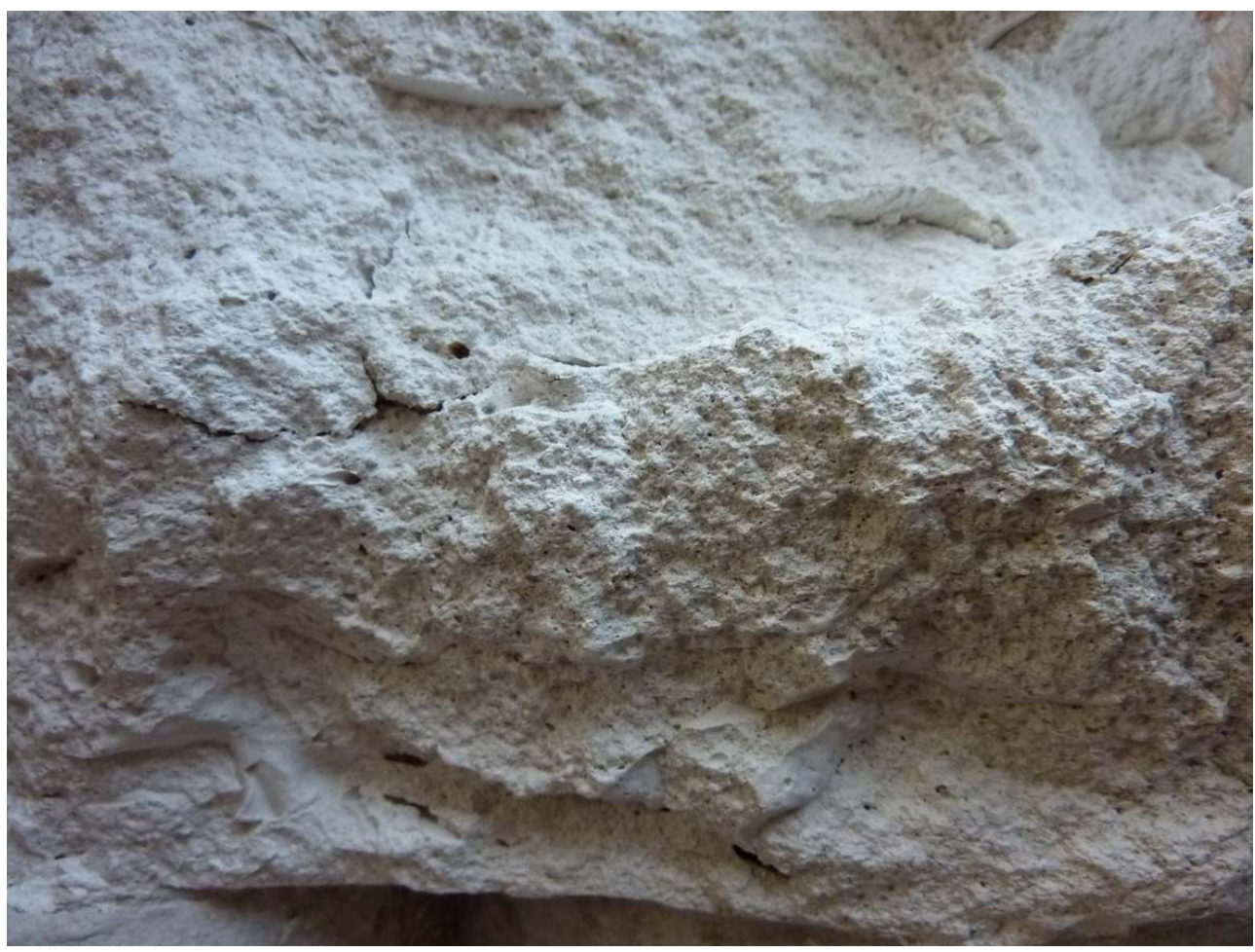

Plâtre d'aspect grossier observé sur un plan de cassure du tirage de l'Hercule-Commode, conservé dans la réserve des tirages de la villa Médicis.

Phot. Roumégoux, P. (c) Pascale Roumégoux.

24 Les tirages intégraux de la Junon Cesi, de L'Apollon du Belvédère et de l'Hercule-Commode (fig. 17) sont lourds et constitués de sections horizontales superposées, tirées en épaisseur, parfois pleines et lestées (briques ou pierres). De plus, les deux derniers sont renforcés d'armatures métalliques épaisses en fer forgé (fig. 18). L'ensemble de ces caractéristiques rappelle le profil des tirages anciens conservés dans la gypsothèque du musée du Louvre ${ }^{56}$. Ce parallèle amène donc à s'interroger sur leur possible appartenance au fonds ancien de la collection italienne, située entre la fin du XVII ${ }^{\mathrm{e}}$ et le cours du XVIII siècle $\mathrm{e}^{57}$. 


\section{Figure 17}

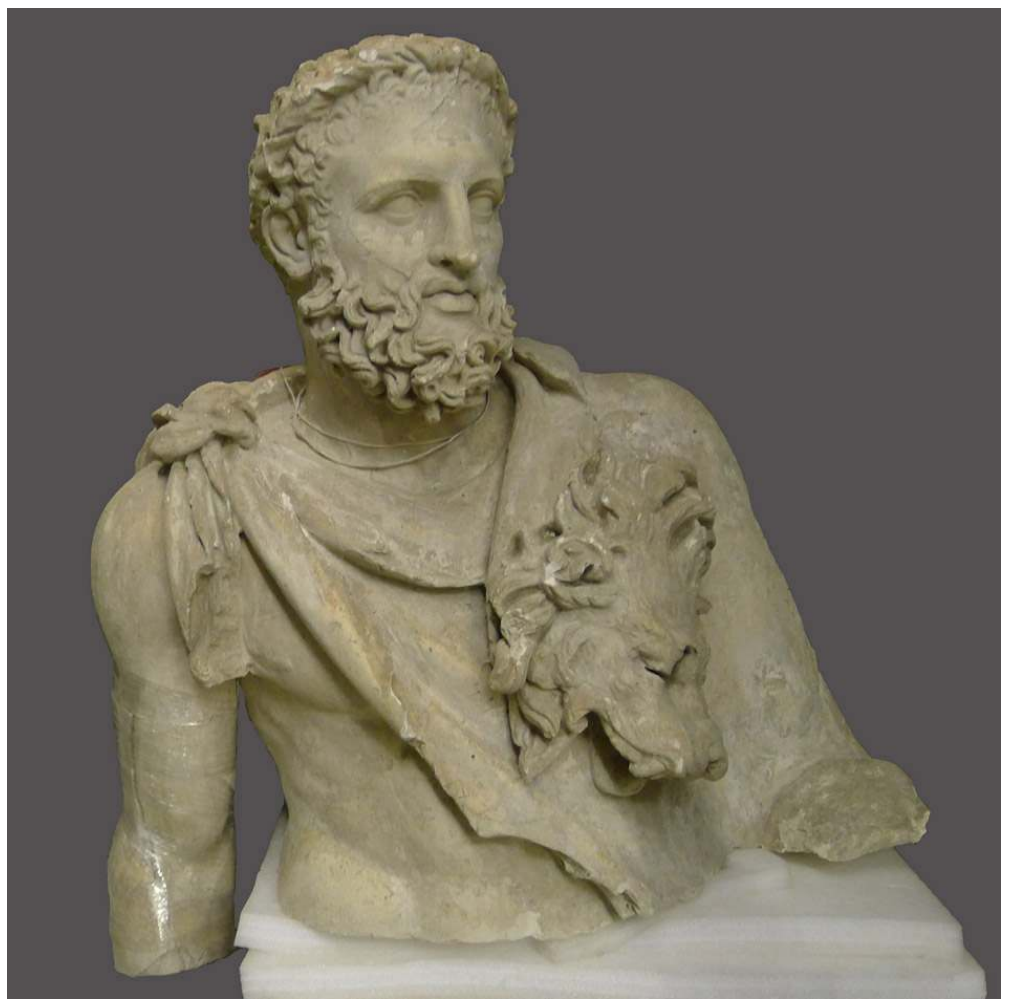

Partie supérieure du tirage intégral de l'Hercule-Commode, conservé dans la réserve des tirages de la villa Médicis.

Phot. Roumégoux, P. (c) Pascale Roumégoux. 
Figure 18

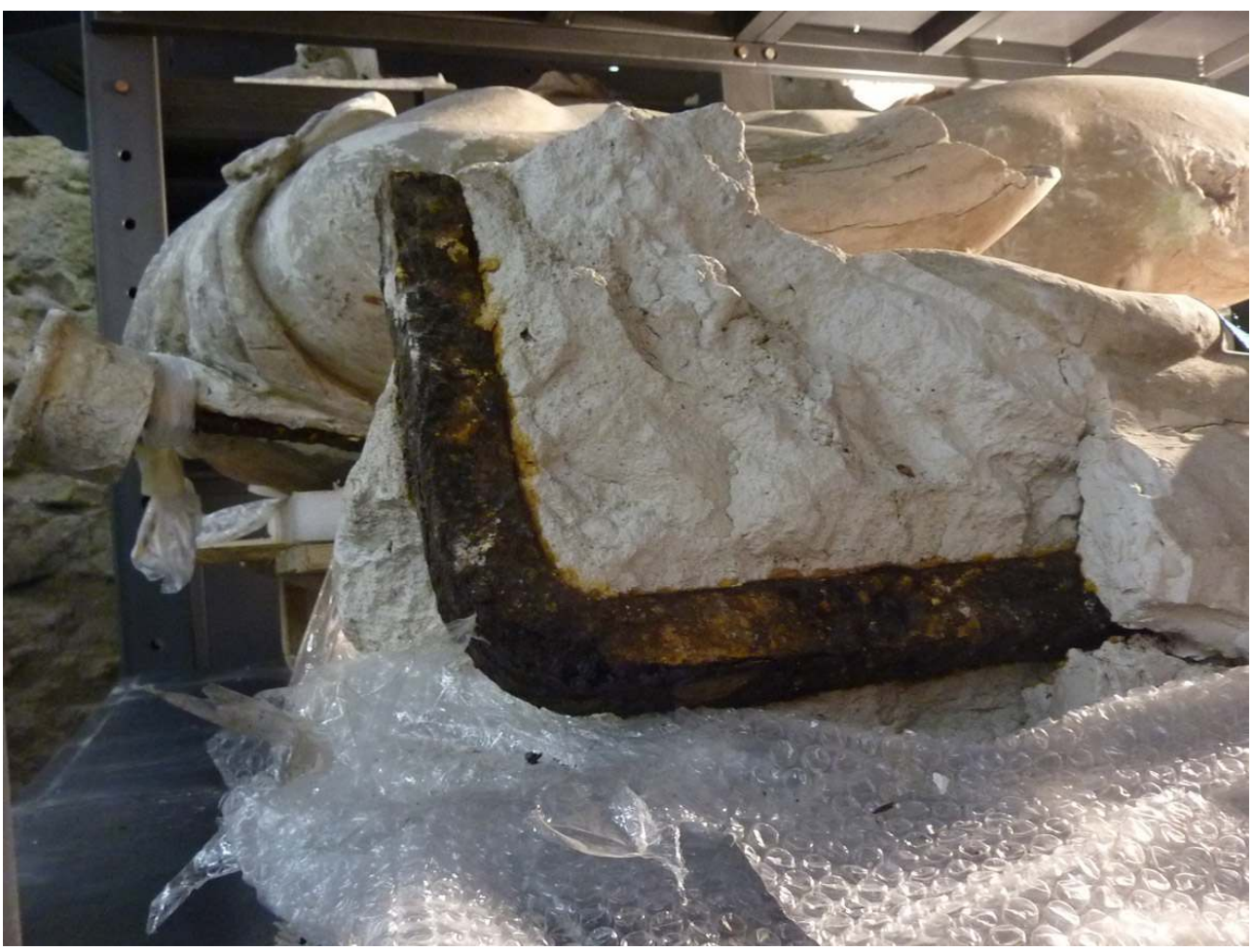

Armature forgée localisée dans la terrasse du tirage intégral de l'Apollon du Belvédère, conservé dans la réserve des tirages de la villa Médicis.

Phot. Roumégoux, P. (C) Pascale Roumégoux.

L'exemple de l'Hercule-Commode abonde dans ce sens. En effet, il présente une technique de tirage parfaitement comparable avec une autre épreuve de l'Hercule-Commode conservée dans la collection du Louvre, datée de la fin du $\mathrm{XvII}^{\mathrm{e}}$ siècle $^{58}$. L'étude comparative des deux réseaux de coutures de ces œuvres laisse supposer que ces deux tirages sont issus du même moule estampé à la terre (fig. 19, fig. 20). Cette dernière donnée apporte un nouvel axe de recherche sur l'histoire de cette œuvre à la fin du XVII ${ }^{e}$ siècle, au sein de l'Académie de France à Rome ${ }^{59}$. 


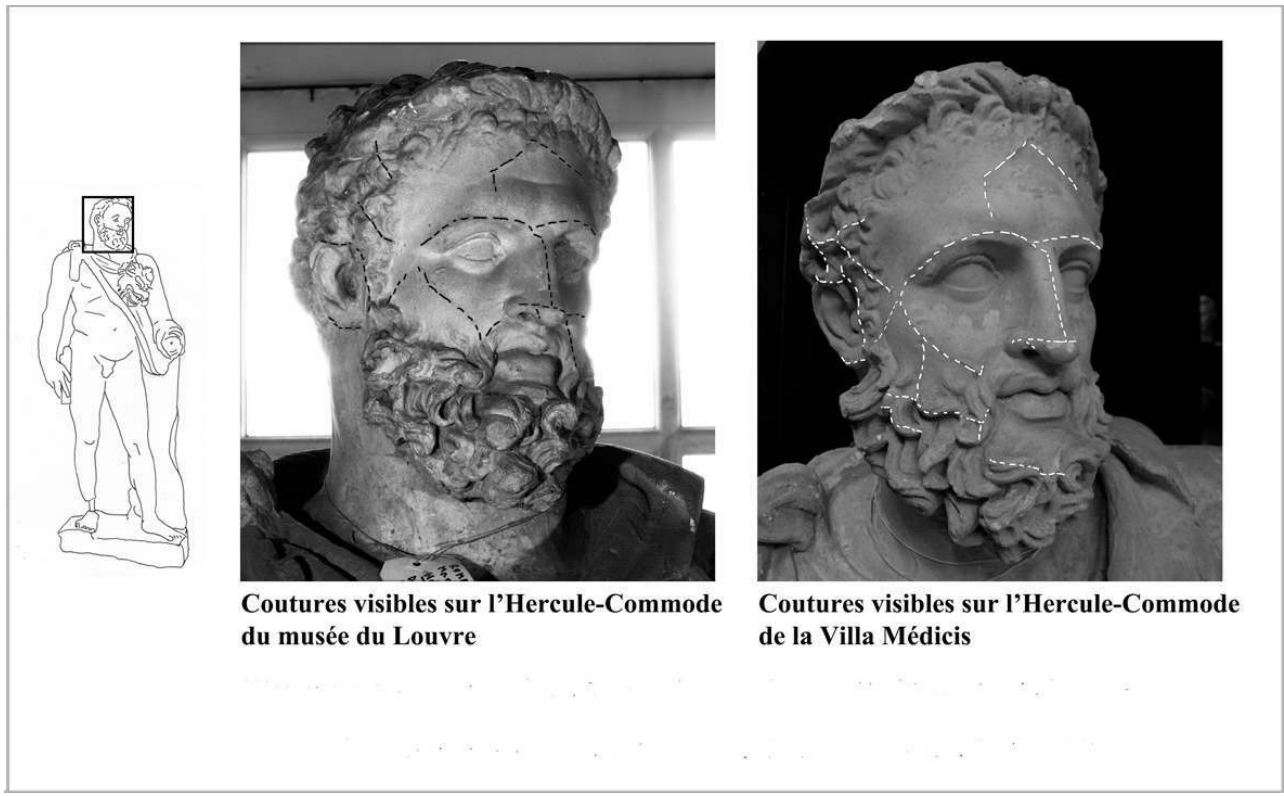

Comparaison des réseaux de coutures des tirages de l'Hercule-Commode conservés à la villa Médicis et à la gypsothèque du musée du Louvre.

Phot. Roumégoux, P. (c) Pascale Roumégoux.

Figure 20

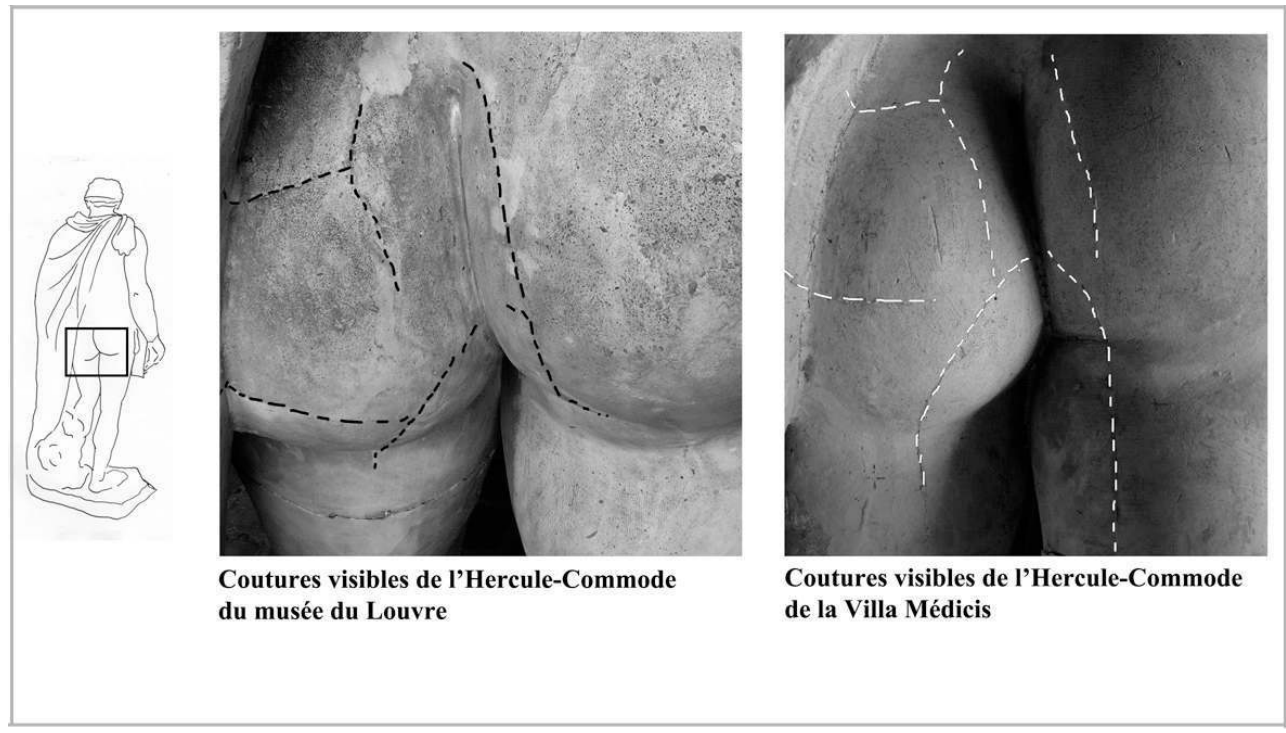

Comparaison des réseaux de coutures des tirages de l'Hercule-Commode conservés à la villa Médicis et à la gypsothèque du musée du Louvre.

Phot. Roumégoux, P. (c) Pascale Roumégoux.

\section{Particularités italiennes}

Il est intéressant de constater que les œuvres présumées appartenir au fonds ancien de cette collection semblent toutes issues de moules à pièces estampés à la terre. Ce même 
constat s'applique aux tirages anciens de la collection du musée du Louvre ${ }^{60}$. Cependant, il est important de préciser qu'un procédé de moulage ne constitue pas en soi un élément de datation. En atteste l'étude menée sur une épreuve de L'Hercule Farnèse conservé à Madrid, daté du XVII ${ }^{e}$ siècle, qui selon ses auteurs aurait été tirée dans un moule à boncreux à pièces en plâtre ${ }^{61}$. Par ailleurs, plusieurs tirages d'éléments architecturaux de la collection de l'Académie, eux-mêmes issus d'estampage, sont datés du XIX ${ }^{e}$ siècle $^{62}$.

Ainsi, les procédés de moulage identifiés sur la collection de la Villa Médicis sont connus et pratiqués depuis plusieurs siècles. Cette continuité technique ressort de l'étude comparative des manuels anciens ${ }^{63}$. Cependant, les observations menées au cours de notre étude, confrontées aux connaissances acquises sur les collections françaises, permettent de distinguer certaines particularités techniques italiennes. En effet, il est important de souligner la dextérité des mouleurs italiens lors de la réalisation des prises d'empreinte à la terre crue ${ }^{64}$. La restitution fidèle des volumes complexes et la nervosité des creux ont parfois nécessité la confection de nombreuses pièces précises. Les coutures visibles sont alors relativement nombreuses et peuvent présenter un profil peu irrégulier, pouvant entraîner une confusion avec le réseau d'un moule à bon-creux à pièces en plâtre par exemple.

Les épreuves du XIX ${ }^{e}$ siècle et $d u x^{e}$ siècle sont également remarquables pour leur finesse de tirage, pouvant mesurer moins d'un centimètre d'épaisseur (fig. 21). Il semble que cela s'explique par la pratique du tirage à la volée qui « permet de tourner les creux lorsque le plâtre est coulé. C'est la façon ordinaire des mouleurs italiens et de là vient qu'ils font des figures si minces ${ }^{65}$. Plusieurs de ces épreuves ne sont pas armées dans la masse de filasse ${ }^{66}$, cette fibre végétale largement employée durant le $\mathrm{XIX}^{\mathrm{e}}$ siècle et le $\mathrm{XX}^{\mathrm{e}}$ siècle en France. La présence de toile de jute n'a également quasiment pas été relevée. En résultent des tirages très légers mais souvent fragiles. 
Figure 21

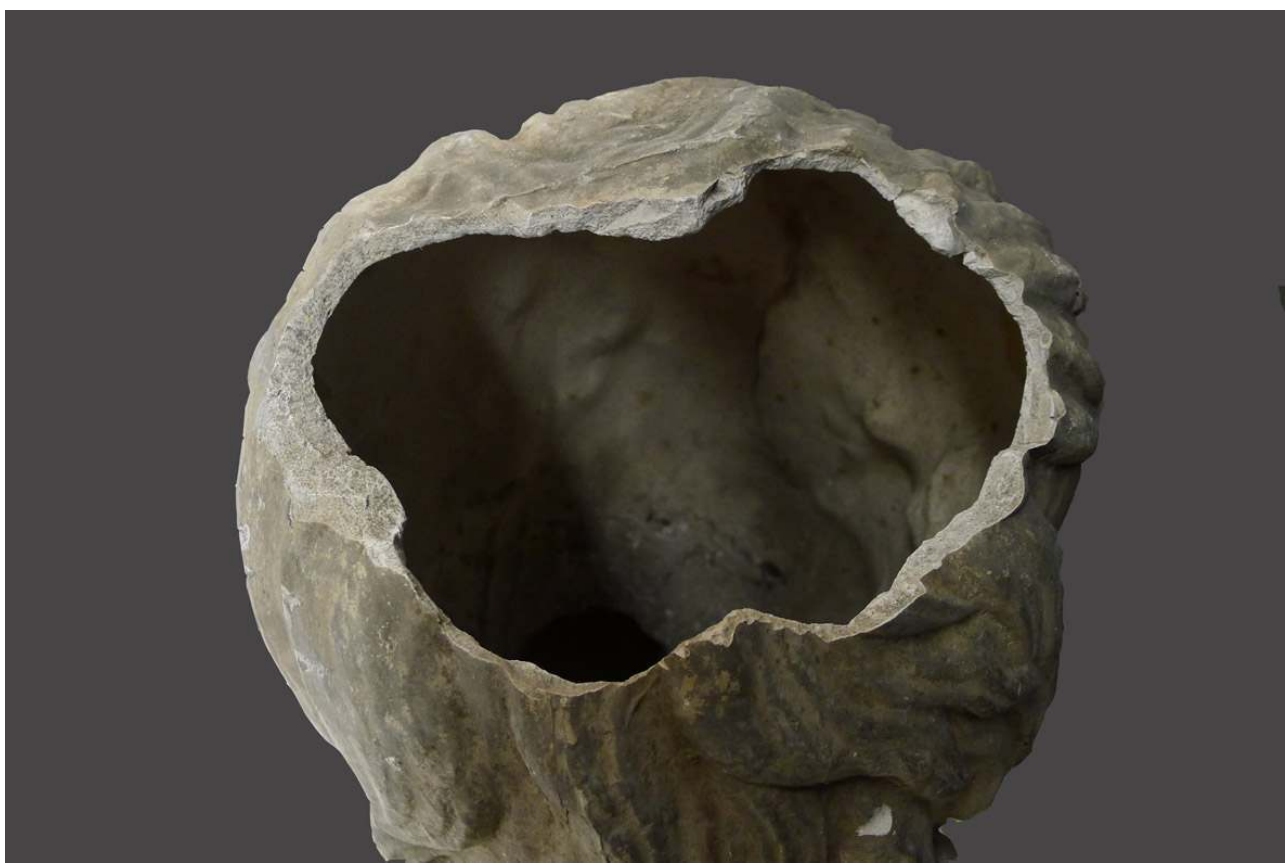

Finesse du tirage d'un buste d'Euripide (NIP0084), conservé dans la réserve des tirages de la villa Médicis.

Phot. Roumégoux, P. (c) Pascale Roumégoux.

Enfin, contrairement à plusieurs tirages datés du XVII ${ }^{\mathrm{e}}$ et XVIII ${ }^{\mathrm{e}}$ siècle du musée du Louvre ${ }^{67}$, aucune impureté visible à l'œil nu, type charbon de bois ou cristaux de gypse non cuit, n'a pu être observée au niveau des plans de cassure des œuvres pouvant appartenir au fonds ancien de cette collection. L'attention particulière portée à certaines épreuves pourrait expliquer un tel constat, comme cela a été avéré pour le tirage de l'Hercule Farnèse conservé à Madrid ${ }^{68}$.

La présentation synthétique de cette étude menée sur la collection de la villa Médicis révèle l'intérêt de confronter les données techniques collectées sur les collections de tirages des diverses institutions françaises et européennes. L'apparente continuité technique dans les procédés de moulage n'exclut pas cependant quelques divergences en fonction du praticien, de l'atelier de moulage, ou encore du pays d'origine. Riches d'informations, ces données ne feront pleinement sens qu'en étant complétées par l'analyse des historiens de l'art et des chercheurs afin d'éclairer le parcours souvent complexe de ces œuvres en plâtre chargées d'Histoire(s).

\section{Biographie}

Pascale Roumégoux est restauratrice de sculptures, diplômée du cycle Conservation-restauration des œuvres sculptées, ESBAT, Tours. Elle est spécialisée en travaux aériens sur cordes en conservation restauration. Elle a été pensionnaire de l'Académie de France à Rome, Villa Médicis, de 2013 à 2014. 


\section{BIBLIOGRAPHIE}

BAYARD, Marc et DI MATTÉO, Colette. « La nouvelle gypsothèque de la villa Médicis et la collection des plâtres ». Studiolo, 8, 2010, p. 308-319.

BAUDRY, Marie-Thérèse (dir.). Sculpture : méthode et vocabulaire. Paris : Éditions du Patrimoine, $6^{\mathrm{e}}$ éd., 2005.

CHEVILLOT, Catherine. «Un traité sur l'art du mouleur en plâtre en 1780 ». Dans BRESC-

BAUTIER, Geneviève (dir.) La sculpture en Occident : études offertes à Jean-René Gaborit. Dijon : éd. Faton, 2007, p. 248-251.

COUSIN DE CONTAMINE. Éloge historique de M. Coustou l'aîné, sculpteur ordinaire du roy et recteur de l'Académie royale de peinture et de sculpture. Paris : Huart, 1737.

DÉLIVRÉ, Jean. «La collection des plâtres de la Villa Médicis ». Science et technologie de la conservation et de la restauration des cuvres du patrimoine (STCR), 2, septembre 1991, p. 103-111.

DÉLIVRÉ, Jean. « La colonne Trajane. Ses moulages (à suivre) ». Annuaire 86/87 Villa Medici. Rome : éd. Carte Segrete, 1987.

FIQUET, M. « L'art du mouleur en plâtre ». Dans BERTRAND, Élie. Description des Arts et Métiers faites ou approuvées par Messieurs de l'Académie des sciences de Paris. Neuchâtel : impr. de la Société typographique, 1780, t. XIV, p. 571-614, p. 587.

FREDERIKSEN, Rune. « The restoration of Two Plaster Casts Acquired by Velasquez in the Seventeenth Century : the Hercules and Flora Farnèse ». Dans MARCHAND, Eckart, FREDERIKSEN, Rune (dir.). Plaster Casts: Making, Collecting and Displaying from Classical Antiquity to the Present. Berlin : de Gruyter, 2010, p. 387-388.

LE BRETON, Élisabeth. «Ingres et les envois de moulages d'après l'antique à l'école royale des Beaux-Arts de Paris ». Dans PICARD-CAJAN, Pascale (dir.). Ingres et l'Antique, l'illusion grecque. Cat. expo. Montauban, musée Ingres, 15 juin-15 septembre, Arles, musée de l'Arles et de la Provence antiques. Arles : Actes sud, 2006, p. 148-165.

LE BRETON, Élisabeth. «Les tirages en plâtre du XVII ${ }^{\mathrm{e}}$ siècle dans la gypsothèque du musée du Louvre. Apport des restaurations récentes ». Monuments et mémoires de la fondation Eugène Piot, t. 92, 2013, p. 67-92.

LEBRUN, M. Nouveau manuel complet du mouleur. Paris : Librairie encyclopédique Roret, coll. « Manuels Roret », 1850.

MARAL, Alexandre, ROUMÉGOUX, Pascale. «À propos de l'Hercule de Coustou de Versailles : découverte d'un plâtre inédit à Rome ». Studiolo, $n^{\circ} 11,2014$, p. 282-289.

PINATEL, Christiane. «Les envois de moulages d'Antique à l'École des Beaux-Arts de Paris par l'Académie de France à Rome ». Dans LAVAGNE, Henri et QUEYREL, François (éd.). Les moulages de sculptures antiques et l'histoire de l'archéologie. Actes du colloque international, Paris, 24 octobre 1997. Genève/Paris : Droz/Champion, p. 75-120.

Manuel Roret, Mouleur en plâtre, etc., de 1829 à 1901.

ROUMÉGOUX, Pascale. «Étude technique de la collection des tirages en plâtre de la Villa Médicis. Chronique d'une pensionnaire restauratrice». Studiolo, $\mathrm{n}^{\circ}$ 11, 2014, p. 270-281. 
SUVÉE, Joseph-Benoît. Correspondance des directeurs de l'Académie de France à Rome. Nouvelle série, 2, Directorat de Suvée (1795-1807). Éd. Georges Brunel et Isabelle Julia. Rome : Ed. dell'Elefante, 1984, t. I, vol. 2, p. 29-28.

\section{Bibliographie en ligne}

Jean-Marc Hofman, « Camille Enlart s'en va-t-en guerre. Le musée de Sculpture comparée pendant la Première Guerre mondiale », In Situ [En ligne], 23 | 2014, mis en ligne le 21 février 2014, consulté le 22 février 2016. URL : http://insitu.revues.org/10894 ; DOI : 10.4000/ insitu.10894.

\section{NOTES}

1. - Sont désignées sous les termes de tirages ou épreuves les œuvres en plâtre obtenues par le procédé de moulage.

2. - Un exposé complet des hypothèses élaborées au cours de cette étude est présenté dans un article bilan : ROUMÉGOUX, Pascale. «Étude technique de la collection des tirages en plâtre de la Villa Médicis. Chronique d'une pensionnaire restauratrice ». Studiolo, $\mathrm{n}^{\circ} 11,2014$, p. 270-281.

3. - La résidence s'est déroulée de mars 2013 à mars 2014.

4. - Études préalables et interventions en restauration sur les collections de la gypsothèque du musée du Louvre, de l'université Paul Valéry de Montpellier, de la collection du musée des Beaux-Arts de Nantes, etc.

5. - L'ensemble des données techniques collectées à la surface des œuvres a été rassemblé dans une base de données gérée par le logiciel Filemaker ${ }^{\circledast}$ conçue par le département des Antiquités grecques, étrusques et romaines du musée du Louvre.

6. - Les observations ont été complétées, dans un deuxième temps, par la consultation de la documentation technique telle que : FIQUET, M. «L'art du mouleur en plâtre ». Dans BERTRAND, Élie. Description des Arts et Métiers faites ou approuvées par Messieurs de l'Académie des sciences de Paris. Neuchâtel : impr. de la Société typographique, 1780, t. XIV, p. 571-614, et les divers Manuels Roret, Mouleur en plâtre, de 1829 à 1901, ainsi que par la rencontre de plusieurs professionnels : Arielle Lebrun (chef de l'atelier de moulage de la Réunion des musées nationaux), Béatrice Kostner et Jacques Bourgeois, mouleurs et sculpteurs.

7. - Il existe diverses publications portant sur l'histoire générale de la collection ou sur l'étude de certains tirages. De plus, un comité de recherche a été formé en 2013 pour poursuivre et approfondir l'étude de cette collection. Il est constitué d'Antoinette Le Normand Romain, Colette di Matteo, Catherine Chevillot, Élisabeth Le Breton et Claire Barbillon, et est piloté par le département d'Histoire de l'art de l'Académie de France à Rome.

8. - BAYARD, Marc et DI MATTÉO, Colette. «La nouvelle gypsothèque de la Villa Médicis et la collection des plâtres ». Studiolo, $\mathrm{n}^{\circ} 8,2010$, p. 308-319.

9. - DÉLIVRÉ, Jean. «La collection des plâtres de la Villa Médicis ». Science et technologie de la conservation et de la restauration des cuvres du patrimoine (STCR), 2, septembre 1991, p. 103-111.

10. - Balthasar Kłossowski, dit Balthus (1908-2001), fut directeur de l'Académie de France à Rome de 1961 à 1977.

11. - La gypsothèque de la villa Médicis a été inaugurée en 2009 par Frédéric Mitterrand, alors directeur de l'Académie. BAYARD, M. et DI MATTÉO, C., 2010, op. cit. Aujourd'hui, cent vingt tirages sont présentés dans la gypsothèque et une soixantaine est conservée dans diverses salles de la villa.

12. - Ce projet de sauvetage de la collection a pu être mené à bien grâce à la collaboration du directeur de la villa Médicis Éric de Chassey, d'Annick Lemoine, Colette Di Matteo, Alessandra Gariazzo, Pauline Pernet, Didier Repellin, Francoise Laurent, du directeur du musée du Louvre 
Jean-Luc Martinez, d'Élisabeth Le Breton et des restaurateurs tels que Jean Délivré et la société De Cesaris.

13. - Actuellement, soixante-douze reliefs sont présentés dans la gypsothèque de la villa Médicis. 14. - Le moulage des reliefs de la colonne Trajane semble se répartir en deux campagnes différentes de prise d'empreinte, l'une au XVII ${ }^{\mathrm{e}}$ siècle et l'autre au XIX ${ }^{\mathrm{e}}$ siècle. Ces datations sont fondées sur la confrontation des données publiées par Jean Délivré et les observations menées au cours de cette étude. Voir: DÉLIVRÉ, Jean. «La colonne Trajane. Ses moulages (à suivre)». Annuaire 86/87 Villa Medici. Rome : éd. Carte Segrete, 1987.

15. - Dans le cas des reliefs de la colonne Trajane, des badigeons successifs ont été appliqués à la surface des œuvres à des dates indéterminées. En 2012, ils ont fait l'objet d'une restauration par la société De Cesaris grâce à laquelle ces badigeons ont pu être dégagés, permettant une meilleure lisibilité de la surface.

16. - Un constat sanitaire succinct (surface et structure) a été également réalisé au cours de cette étude et renseigné dans la base de données.

17. - La filasse, constituée de fibres végétales type lin ou chanvre, est employée pour armer les tirages dans la masse et maintenir les armatures internes. Elle est d'usage courant au XIX ${ }^{\mathrm{e}}$ siècle, permettant l'obtention de tirages de fine épaisseur, légers et résistants. Durant nos recherches, son usage dans la masse n'a pas pu être avéré avant le XIX ${ }^{\mathrm{e}}$ siècle.

18. - Gâchage : fait de délayer du plâtre dans l'eau et de le malaxer.

19. - Ces traces ne sont malheureusement plus toujours visibles à la surface des œuvres de la collection : plus de trente tirages ont été poncés ou repeints, plus d'une dizaine présentent une surface dissoute du fait d'une exposition en extérieur. Enfin, le réseau de coutures de quatrevingt-dix-huit tirages a été reparé, c'est-à-dire dissimulé à l'outil, rendant impossible la lecture des informations superficielles.

20. - Moule ou creux: ensemble des éléments en matériaux appropriés (plâtre, gélatine, terre, entre autres) délimitant une empreinte destinée à recevoir une substance à l'état liquide ou pâteux qui, après solidification, prend la forme de cette empreinte et reproduit la sculpture qui a servi de modèle. Voir: BAUDRY, Marie-Thérèse (dir.). Sculpture: méthode et vocabulaire. Paris : Éditions du Patrimoine, $6^{\mathrm{e}}$ éd., 2005, p. 562.

21. - Moulage à bon-creux: procédé de moulage qui consiste à faire un moule réutilisable dans lequel on peut façonner un nombre plus ou moins important d'épreuves (épreuve de série). Moulage par estampage : procédé de moulage à creux-perdu, qui s'effectue en pressant de la terre molle ou du mastic sur toute la surface d'une sculpture en matériau dur (marbre, pierre, bois). On obtient une épreuve d'estampage. Moulage à creux-perdu : procédé de moulage qui consiste à faire un moule qui doit être détruit pour permettre l'extraction de l'épreuve qu'il contient (épreuve unique : nommée épreuve originale) Voir : BAUDRY, M.-Th., op. cit., p. 558.

22. - Cent vingt-quatre épreuves ont pu être identifiées comme pouvant être issues d'un moule à bon-creux en plâtre.

23. - LEBRUN, M. Nouveau manuel complet du mouleur. Paris : Librairie encyclopédique Roret, coll. «Manuels Roret », 1850, p. 34.

24. - Pallas de Velletri, NIP0006, conservé dans la gypsothèque de la villa Médicis. Le numéro d'inventaire cité correspond à l'inventaire réalisé par Christiane Pinatel, du musée du Louvre, en 1983.

25. - Contre-dépouille : légère pente présentée par certaine partie d'un modèle qui rend difficile son extraction du moule. On dit d'un modèle qui présente des cavités dont le fond est plus large que l'ouverture qu'il est en contre-dépouille. BAUDRY, M.-Th., op. cit., p. 563.

26. - Information recueillie auprès d'Arielle Lebrun, chef de l'atelier de moulage de la RMN à Paris (Réunion des musées nationaux), Rome, novembre 11/2013.

27. - Dioscure de Montecavallo, NIP0043-II, conservé dans la gypsothèque de la villa Médicis. 
28. - L'utilisation concomitante et ponctuelle de mastic cireux pour la prise d'empreinte de certaines parties délicates ou présentant des cavités importantes ne peut également être exclue. 29. - Hercule-Commode, NIP0147, conservé dans la réserve des tirages de la villa Médicis.

30. - Ces aspérités pourraient s'expliquer par l'application d'un corps gras à la surface de la terre crue avant la coulée du plâtre de tirage, ceci afin de faciliter le démoulage et d'épargner l'empreinte.

31. - « Il est vrai que de ces calques on peut tirer rarement plus de deux ou trois épreuves, encore faut-il opérer vite [...] ». Archives de l'Académie de France à Rome (AFR), carton 37, fol. 392, lettre manuscrite de Paul Lemoyne (Paris, 1784-Rome, 1873 ; statuaire, correspondant de l'Institut de France, membre de l'Académie de Saint-Luc depuis 1836) du 4 septembre 1839 à Ingres (directeur de l'AFR).

32. - FIQUET, M., op. cit., p. 580.

33. - Cent vingt-six tirages de la collection semblent issus d'un moule estampé à la terre.

34. - Anacreon, NIP0117, conservé dans la gypsothèque de la villa Médicis.

35. - Parée : mise en forme à l'outil des pièces après l'application de la terre par pression sur le modèle à mouler.

36. - Ce mastic peut être composé de cire, poix, plâtre très fin, etc. La littérature technique fait mention de diverses recettes.

37. - Paul Lemoyne, 1839, document cité n. 31.

38. - Portrait de Ziem, NIP0368, conservé dans la réserve des tirages de la villa Médicis. Victor Joseph Jean Ambroise Ségoffin (1867-1925), pensionnaire de 1897 à 1901.

39. - Ugolin, NIP0, conservé dans la réserve des tirages de la villa Médicis. Jean-Baptiste Carpeaux (1827-1875), pensionnaire de 1856 à 1859.

40. - Ces tirages sont alors obtenus par un procédé de « surmoulage».

41. - À ces quarante-cinq "surtirages » identifiés pourraient s'additionner d'autres tirages dont les réseaux ont été reparés ou des tirages moulés sur des épreuves dont le réseau a été également reparé, n'apparaîtrait alors qu'un seul réseau, troublant l'information.

42. - Il semble que pour la réalisation de moules à pièces en terre crue, il était d'usage d'utiliser des outils en bois. Ces outils épargnaient sans doute davantage la surface fragile des plâtres.

43. - La barbotine est constituée d'un mélange d'eau et d'argile crue.

44. - L'envoi de tirages en France est également à prendre en compte. PINATEL, Christiane. «Les envois de moulages d'Antique à l'École des Beaux-Arts de Paris par l'Académie de France à Rome ». Dans LAVAGNE, Henri et QUEYREL, François (éd.). Les moulages de sculptures antiques et l'histoire de l'archéologie. Actes du colloque international, Paris, 24 octobre 1997. Genève/Paris : Droz/Champion, p. 75-120 ; LE BRETON, Élisabeth. «Ingres et les envois de moulages d'après l'antique à l'école royale des Beaux-Arts de Paris ». Dans PICARD-CAJAN, Pascale (dir.). Ingres et l'Antique, l'illusion grecque. Cat. expo. Montauban, musée Ingres, 15 juin-15 septembre, Arles, musée de l'Arles et de la Provence antiques. Arles : Actes sud, 2006, p. 148-165.

45. - Démosthène, NIP0143, conservé dans la gypsothèque de la villa Médicis.

46. - Hercule Enfant, NIP0157, conservé dans la gypsothèque de la villa Médicis.

47. - « Les moulages entrés sous le directorat d'Albert Besnard font partie de l'exposition de moulages de sculpture française inaugurée le 10 juin 1919 à des fins de propagande et d'histoire de l'art». Jean-Marc Hofman, "Camille Enlart s'en va-t-en guerre. Le musée de Sculpture comparée pendant la Première Guerre mondiale », In Situ [En ligne], 23 | 2014, mis en ligne le 21 février 2014, consulté le 22 février 2016. URL : http://insitu.revues.org/10894; DOI : 10.4000/ insitu.10894

48. - La pratique de mise aux points par cadre ou "dessous châssis » ne peut également être exclue. 
49. - SUVÉE, Joseph-Benoît. Correspondance des directeurs de l'Académie de France à Rome. Nouvelle série, 2, Directorat de Suvée (1795-1807). Éd. Georges Brunel et Isabelle Julia. Rome : Ed. dell'Elefante, 1984 , t. I, vol. 2, p. 29-28.

50. - D'après la littérature, il apparaît que certains pensionnaires sculpteurs s'accordaient une certaine liberté lors de la réalisation des copies des chefs-d'œuvre antiques. COUSIN DE CONTAMINE. Éloge historique de M. Coustou l'aîné, sculpteur ordinaire du roy et recteur de l'Académie royale de peinture et de sculpture. Paris : Huart, 1737, p. 4-5.

51. - Hercule Enfant, NIP0327, conservé dans la réserve des tirages de la villa Médicis.

52. - Junon Cesi, NIP0146, conservé dans la réserve des tirages de la villa Médicis.

53. - L'Apollon du Belvédère, NIP0082, conservé dans la réserve des tirages de la villa Médicis.

54. - Des tests complémentaires ont également été réalisés sur des prélèvements avec de l'acide chlorhydrique. Ils nous ont permis d'appréhender la pureté du plâtre de tirage, et de déceler l'éventuelle présence de résidus carbonatés ou l'ajout de charge calcaire; les résultats obtenus ont été reportés dans la fiche «Étude technique » de la base.

55. - LE BRETON, É., art. cit.

56. - Ibid.

57. - Une liste plus complète d'œuvres singulières susceptibles d'appartenir au fonds ancien de la collection est donnée dans l'article général. ROUMÉGOUX, P., art. cit.

58. - Paris, musée du Louvre, département des Antiquités grecques, étrusques et romaines, collection des tirages en plâtre conservée dans les Petites Écuries du château de Versailles, Gy $0247(220 \times 94 \times 60 \mathrm{~cm})$. Tirage du XVII ${ }^{\mathrm{e}}$ siècle. LE BRETON, É., art. cit.

59. - MARAL, Alexandre, ROUMÉGOUX, Pascale. « À propos de l'Hercule de Coustou de Versailles : découverte d'un plâtre inédit à Rome ». Studiolo, $n^{\circ} 11,2014$.

60. - LE BRETON, É., art. cit.

61. - FREDERIKSEN, Rune. "The restoration of Two Plaster Casts Acquired by Velasquez in the Seventeenth Century : the Hercules and Flora Farnèse ». Dans MARCHAND, Eckart, FREDERIKSEN, Rune (dir.). Plaster Casts: Making, Collecting and Displaying from Classical Antiquity to the Present. Berlin : de Gruyter, 2010, p. 387-388.

62. - Les tirages architecturaux ont été intégrés dans la collection de la villa à partir du directorat de Pierre Adrien Pâris (1807) ; information recueillie auprès d'Alessandra Gariazzo, responsable des archives, villa Médicis.

63. - CHEVILLOT, Catherine. "Un traité sur l'art du mouleur en plâtre en 1780 ». Dans BRESCBAUTIER, Geneviève (dir.) La sculpture en Occident : études offertes à Jean-René Gaborit. Dijon : éd. Faton, 2007, p. 248-251. Consultation des diverses sources bibliographiques : FIQUET, M., op. cit. et manuels Roret de 1829 à 1901.

64. - « Les mouleurs italiens n'ont pas d'égaux dans cette espèce de moulage ». P. Lemoyne, 1839, document cité n. 31 .

65. - FIQUET, M., op. cit., p. 587.

66. - La filasse, sous forme de polochons enduits de plâtre, est plus fréquemment observée pour maintenir les armatures internes.

67. - LE BRETON, É., art. cit.

68. - L'étude du tirage de l'Hercule Farnèse déjà évoqué, daté du $\mathrm{XVII}^{\mathrm{e}}$ siècle et conservé à Madrid, menée en 2006, indique qu'un soin particulier pouvait être apporté dans la fabrication du plâtre de tirage afin d'obtenir une épreuve de haute qualité. FREDERIKSEN, Rune, art. cit., p. 400. 


\section{RÉSUMÉS}

Les diverses traces observées à la surface des épreuves en plâtre conservées à l'Académie de France à Rome ont été des supports de réflexion sur les diverses techniques de moulage utilisées au cours des siècles sur cette collection chargée d'Histoire.

The various traces that can be seen on the surface of the plaster models held today by the French Academy at Rome (Villa Médicis) are the occasion for some thinking about the various moulding techniques used in the past for this collection, rich in historical references.moulage, tirage, plâtre, épreuve, étude technique, Académie de France à Rome, Villa Médicis, gypsothèque, moule, coutures

\section{INDEX}

Mots-clés : moulage, tirage, plâtre, épreuve, étude technique, Académie de France à Rome, Villa Médicis, gypsothèque, moule, coutures

Keywords : plaster cast, edition, plaster, proof copy, technical study, French Academy at Rome, Villa Médicis, gypsotheque, mould, seams

\section{AUTEUR}

\section{PASCALE ROUMÉGOUX}

Restauratrice de sculptures, Pensionnaire de l'Académie de France à Rome, Villa Médicis, 2013-2014 roumegouxpascale@yahoo.fr 